\title{
Fault Detection for Multimodal Process Using Quality-Relevant Kernel Neighborhood Preserving Embedding
}

\author{
Yunpeng Fan, Wenyou Du, Yingwei Zhang, and Xiaogang Wang \\ State Key Laboratory of Synthetical Automation for Process Industries, Northeastern University, Liaoning 100819, China \\ Correspondence should be addressed to Yingwei Zhang; zhangyingwei@mail.neu.edu.cn
}

Received 18 May 2015; Accepted 6 August 2015

Academic Editor: Xinggang Yan

Copyright (C) 2015 Yunpeng Fan et al. This is an open access article distributed under the Creative Commons Attribution License, which permits unrestricted use, distribution, and reproduction in any medium, provided the original work is properly cited.

\begin{abstract}
A new method named quality-relevant kernel neighborhood preserving embedding (QKNPE) has been proposed. Quality variables have been considered for the first time in kernel neighborhood preserving embedding (KNPE) method for monitoring multimodal process. In summary, the whole algorithm is a two-step process: first, to improve manifold structure and to deal with multimodal nonlinearity problem, the neighborhood preserving embedding technique is introduced; and second to monitoring the complete production process, the product quality variables are added in the objective function. Compared with the conventional monitoring method, the proposed method has the following advantages: (1) the hidden manifold which related to the character of industrial process has been embedded to a low dimensional space and the identifying information of the different mode of the monitored system has been extracted; (2) the product quality as an important factor has been considered for the first time in manifold method. In the experiment section, we applied this method to electrofused magnesia furnace (EFMF) process, which is a representative case study. The experimental results show the effectiveness of the proposed method.
\end{abstract}

\section{Introduction}

Product quality monitoring has attracted a lot of attention like process monitoring in nearly two decades. Scholars and enterprises have made great efforts and obtained many worthy achievements [1]. Data-based process monitoring is an important means in solving some such issue [2-4]. Typical data-based process monitoring methods are used to deal with the input variable space, such as principal component analysis (KPCA) [5-7] or independent component analysis (KICA) [8-10]. Partial least squares (PLS) [11-14] or kernel partial least squares (KPLS) [15-18] can build the model of input/process variables and output/quality variables; however, the effect is not ideal for multimodal process $[19,20]$. Because root causes of potential quality problems are in process variables [21], synthetically considering the whole variables is a subject worthy of study.

Large-scale data can be obtained and stored from industrial process, which contain process variables and quality variables [22-24]. Storing and processing these received data are a difficult problem; we need the mathematical transformation and dimensionality reduction. Moreover, because process variable is usually measured directly, it does not cause big time delay generally. However, quality variable is measured by many complex means, such as chemical test; it will cause big time delay. Sample frequencies of process variables and quality variables are usually not synchronized [21]. This problem may increase the false alarm rate and missed alarm rate in the monitoring of multimodal process using the above traditional method. Manifold-based method, such as locally linear embedding [25-28], is a kind of method which keeps relative relationship among the data points from a high dimension space to a low dimension space. As a manifold learning framework, it calculates the Euclidean distance between every data point and its neighbors and then keeps this relationship to a lower dimensional manifold in the process of dimension reduction. Time serials of sample will not affect the effectiveness of the previous mentioned method. 


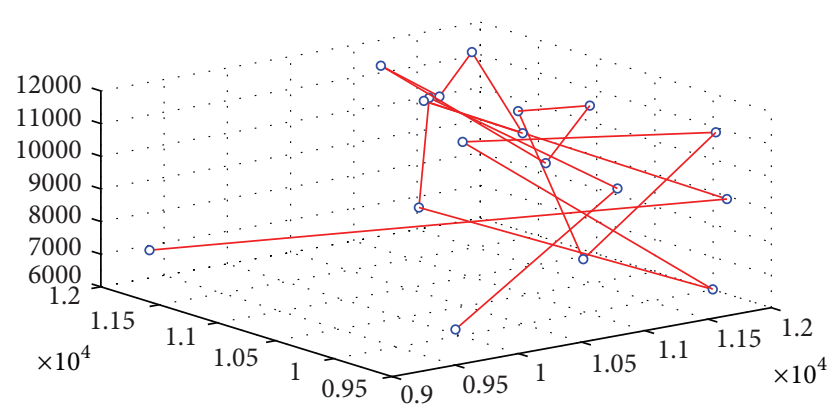

FIGURE 1: Data nonlinear relationship of EFMF.

However, for strong-nonlinearity problems, it may not be used directly, because some data points may lay on nonmanifold surfaces. In this case, kernel technique was introduced [20], which projects the raw data into a higher dimensional linear space, that is, the feature space. The manifold structure of data is improved by this transformation. In addition, since change of feed, adjustment of production plan, and switching set value by human, industrial production processes have multimodal characteristics $[29,30]$. The traditional monitoring methods are not available for multimodal processes monitoring as previously stated. The quality variables of each mode and the product quality variables were unnoticed in the existing manifold-based monitoring methods.

This paper proposed a new manifold-based monitoring method used to detect fault in multimodal process, which considered the quality variables for the first time. We can decompose the process into two steps as follows: first, using KNPE technique to improve manifold structure and to deal with multimodal nonlinearity problem; second, adding preprocessed quality variables in the objective function to monitoring the complete production process. The proposed method has the following advantages: Firstly, the hidden manifold has been embedded in a low dimensional space and the identifying information of the different mode has been extracted. Secondly, the product quality has been considered in manifold method for the first time. To verify the effectiveness of the proposed method, EFMF is introduced in this paper. EFMF process will be described in the experiment section, which is a typical industrial object with characteristic of multimode and nonlinearity $[31,32]$. Through experimental examples, detection capability of the algorithm proposed will be verified.
The main parts of this paper are organized as follows. In Section 2, KNPE algorithm and statistic are illustrated. In Section 3, we show how QKNPE works in process monitoring. An important example of EFMF process is studied to verify the effectiveness of the proposed method.

\section{Kernel Neighborhood Preserving Embedding Algorithm and Statistic}

2.1. The Theory of Kernel Neighborhood Preserving Embedding Algorithm. Nonlinear characteristics of complex industrial process limit the application of many linear processes monitoring method. The basic principle of kernel function method is as follows. We assume process data after standardization as $\mathbf{X}_{m}=\left[\mathbf{x}_{1}, \mathbf{x}_{2}, \ldots, \mathbf{x}_{n}\right] \in \mathbf{R}^{m \times n}, n$ is sample size obtained by sampling, and $m$ is dimension of measure variable. We use a nonlinear mapping $\Phi(\cdot)$ to map input data into a high dimensional feature space and then process the data in high dimensional feature space. We assume that the data points in the neighborhood fit locally linearly in the feature space. As shown in Figure 1, it can convert the computation of nonlinear high dimensional feature space after inner product operation into kernel function operation of the original data space by kernel function $\mathbf{K}\left(\mathbf{X}_{i}, \mathbf{X}_{j}\right)=\boldsymbol{\Phi}^{T}\left(\mathbf{X}_{i}\right) \boldsymbol{\Phi}\left(\mathbf{X}_{j}\right)$. The kernel function used commonly in process monitoring areas is radial basis kernel function $\mathbf{K}\left(\mathbf{X}_{i}, \mathbf{X}_{j}\right)=\exp \left(-\| \mathbf{X}_{i}-\right.$ $\left.\mathbf{X}_{j} \|^{2} / c\right)$, where $c=r M \sigma^{2}, r$ is parameter, $M$ is the dimensions of the input data, and $\sigma^{2}$ is variance [33]. $\Phi(\mathbf{X})=$ $\left[\boldsymbol{\Phi}\left(\mathbf{x}_{1}\right), \boldsymbol{\Phi}\left(\mathbf{x}_{2}\right), \ldots, \boldsymbol{\Phi}\left(\mathbf{x}_{n}\right)\right] \in \mathbf{R}^{v}$ is data of high dimensional feature space. $v$ is the dimensions of the high dimensional feature space. The Euclidean distance between two points in high dimensional feature space can be calculated according to the following formula:

$$
\begin{aligned}
& \left\|\Phi\left(\mathbf{x}_{i}\right)-\Phi\left(\mathbf{x}_{j}\right)\right\| \\
& =\sqrt{\left(\Phi\left(\mathbf{x}_{i}\right)-\Phi\left(\mathbf{x}_{j}\right)\right)^{T}\left(\Phi\left(\mathbf{x}_{i}\right)-\Phi\left(\mathbf{x}_{j}\right)\right)} \\
& =\sqrt{\Phi^{T}\left(\mathbf{x}_{i}\right) \Phi\left(\mathbf{x}_{i}\right)+\Phi^{T}\left(\mathbf{x}_{j}\right) \Phi\left(\mathbf{x}_{j}\right)-2 \Phi^{T}\left(\mathbf{x}_{i}\right) \Phi\left(\mathbf{x}_{j}\right)} \\
& =\sqrt{\mathbf{K}\left(\mathbf{x}_{i}, \mathbf{x}_{i}\right)+\mathbf{K}\left(\mathbf{x}_{j}, \mathbf{x}_{j}\right)-2 \mathbf{K}\left(\mathbf{x}_{i}, \mathbf{x}_{j}\right)} .
\end{aligned}
$$

And then, the following weight matrix of KNPE W can be calculated according to the following formula [34]:

$$
\begin{array}{ll}
\min & \mathbf{e}(\mathbf{W})=\sum_{i=1}^{n}\left\|\boldsymbol{\Phi}\left(\mathbf{x}_{i}\right)-\sum_{j=1}^{n} \mathbf{W}_{i j} \boldsymbol{\Phi}\left(\mathbf{x}_{j}\right)\right\|^{2}=\sum_{i=1}^{k}\left(\mathbf{K}\left(\mathbf{x}_{i}, \mathbf{x}_{i}\right)-2 \sum_{j=1}^{k} \mathbf{W}_{i j} \mathbf{K}\left(\mathbf{x}_{i}, \mathbf{x}_{j}\right)+\sum_{j=1}^{k} \sum_{j=1}^{k} \mathbf{W}_{i j} \mathbf{W}_{i j} \mathbf{K}\left(\mathbf{x}_{j}, \mathbf{x}_{j}\right)\right) \\
\text { s.t. } \quad & \sum_{j=1}^{n} \mathbf{W}_{i j}=1 \\
& \mathbf{W}_{i j}=0, \quad j \notin J_{i}, i=1, \ldots, n .
\end{array}
$$


Solving (2),

$$
\begin{aligned}
\mathbf{W}_{i j} & =\sum_{i=1}^{n} \mathbf{K}^{-1}\left(\mathbf{x}_{i}, \mathbf{x}_{j}\right)\left(\mathbf{K}\left(\mathbf{x}, \mathbf{x}_{i}\right)\right. \\
& \left.+\frac{1-\sum_{i=1}^{n} \sum_{j=1}^{n} \mathbf{K}^{-1}\left(\mathbf{x}_{i}, \mathbf{x}_{j}\right) \mathbf{K}\left(\mathbf{x}, \mathbf{x}_{i}\right)}{\sum_{i=1}^{n} \sum_{j=1}^{n} \mathbf{K}^{-1}\left(\mathbf{x}_{i}, \mathbf{x}_{j}\right)}\right) .
\end{aligned}
$$

The global public information $\boldsymbol{\Phi}\left(\mathbf{X}_{g}\right)=\left[\Phi\left(\mathbf{x}_{g, 1}\right), \ldots\right.$, $\left.\Phi\left(\mathbf{x}_{g, n}\right)\right] \in \mathbf{R}^{d}$ can be extracted by keeping local structure of the input data. The cost error of global public subspace is defined as

$$
\begin{aligned}
\min & \mathbf{e}\left(\boldsymbol{\Phi}\left(\mathbf{X}_{g}\right)\right)=\sum_{i=1}^{n}\left\|\boldsymbol{\Phi}\left(\mathbf{x}_{g, i}\right)-\sum_{j=1}^{n} \mathbf{W}_{i j} \boldsymbol{\Phi}\left(\mathbf{x}_{g, j}\right)\right\|^{2}=\operatorname{trace}\left(\boldsymbol{\Phi}^{T}\left(\mathbf{X}_{g}\right)-\mathbf{W} \boldsymbol{\Phi}^{T}\left(\mathbf{X}_{g}\right)\right)^{T}\left(\boldsymbol{\Phi}^{T}\left(\mathbf{X}_{g}\right)-\mathbf{W} \boldsymbol{\Phi}^{T}\left(\mathbf{X}_{g}\right)\right) \\
& =\operatorname{trace}\left(\boldsymbol{\Phi}\left(\mathbf{X}_{g}\right) \mathbf{M} \boldsymbol{\Phi}^{T}\left(\mathbf{X}_{g}\right)\right) \\
\text { s.t. } & \boldsymbol{\Phi}^{T}\left(\mathbf{X}_{g}\right) \boldsymbol{\Phi}\left(\mathbf{X}_{g}\right)=\mathbf{I}_{n},
\end{aligned}
$$

where $\mathbf{M}=\mathbf{M}^{T}=(\mathbf{I}-\mathbf{W})^{T}(\mathbf{I}-\mathbf{W})$ and $\mathbf{I}_{n}$ is $n$ order unit matrix.

Using the Lagrange multiplier method to deduce the global public space $\boldsymbol{\Phi}\left(\mathbf{X}_{g}\right)$, the Lagrange function is

$$
\begin{aligned}
L= & \operatorname{trace}\left(\boldsymbol{\Phi}\left(\mathbf{X}_{g}\right) \mathbf{M} \boldsymbol{\Phi}^{T}\left(\mathbf{X}_{g}\right)\right) \\
& -\lambda\left(\boldsymbol{\Phi}^{T}\left(\mathbf{X}_{g}\right) \Phi\left(\mathbf{X}_{g}\right)-\mathbf{I}_{n}\right) .
\end{aligned}
$$

Setting Lagrange function as $\boldsymbol{\Phi}\left(\mathbf{X}_{g}\right)$ and partial derivative of $\lambda$ as 0 , we can obtain

$$
\begin{aligned}
\frac{\partial L}{\partial \Phi\left(\mathbf{X}_{g}\right)} & =\boldsymbol{\Phi}\left(\mathbf{X}_{g}\right) \mathbf{M}+\boldsymbol{\Phi}\left(\mathbf{X}_{g}\right) \mathbf{M}^{T}-2 \lambda \Phi\left(\mathbf{X}_{g}\right) \\
& =2 \Phi\left(\mathbf{X}_{g}\right) \mathbf{M}-2 \lambda \boldsymbol{\Phi}\left(\mathbf{X}_{g}\right)=\mathbf{0}_{d \times n} \\
\frac{\partial L}{\partial \lambda} & =\boldsymbol{\Phi}\left(\mathbf{X}_{g}\right)^{T} \boldsymbol{\Phi}\left(\mathbf{X}_{g}\right)-\mathbf{I}_{n}=\mathbf{0}_{n} .
\end{aligned}
$$

$$
\begin{aligned}
& \min \mathbf{e}(\mathbf{G})=\operatorname{trace}\left(\Phi^{T}(\mathbf{X}) \mathbf{G}-\mathbf{W} \boldsymbol{\Phi}^{T}(\mathbf{X}) \mathbf{G}\right)^{T}\left(\boldsymbol{\Phi}^{T}(\mathbf{X}) \mathbf{G}-\mathbf{W} \boldsymbol{\Phi}^{T}(\mathbf{X}) \mathbf{G}\right)=\operatorname{trace}\left(\mathbf{G}^{T} \boldsymbol{\Phi}(\mathbf{X}) \mathbf{M} \boldsymbol{\Phi}^{T}(\mathbf{X}) \mathbf{G}\right) \\
& \text { s.t. } \quad \mathbf{G G}^{T}=\mathbf{I}_{v},
\end{aligned}
$$

where $\mathbf{G}$ is mapping projection matrix from high dimension space to low dimension space. The constraint condition for the projection mapping matrix is $\mathbf{G}^{T} \mathbf{G}=\mathbf{I}_{d}$. Lagrange multiplier can be used to solve it as follows:

$$
\begin{aligned}
L & =\operatorname{trace}\left(\mathbf{G}^{T} \boldsymbol{\Phi}(\mathbf{X}) \mathbf{M} \boldsymbol{\Phi}^{T}(\mathbf{X}) \mathbf{G}\right)-\lambda\left(\mathbf{G}^{T} \mathbf{G}-\mathbf{I}_{d}\right) \\
\frac{\partial L}{\partial \mathbf{G}} & =\boldsymbol{\Phi}(\mathbf{X}) \mathbf{M} \boldsymbol{\Phi}^{T}(\mathbf{X}) \mathbf{G}+\left(\boldsymbol{\Phi}(\mathbf{X}) \mathbf{M} \boldsymbol{\Phi}^{T}(\mathbf{X})\right)^{T} \mathbf{G}
\end{aligned}
$$

The minimal solution $\boldsymbol{\Phi}\left(\mathbf{X}_{g}\right)$ satisfies this form:

$$
\mathbf{M} \Phi\left(\mathbf{X}_{g}\right)=\lambda \Phi\left(\mathbf{X}_{g}\right)
$$

So the global public space $\boldsymbol{\Phi}\left(\mathbf{X}_{g}\right)$ is the first $d$ minimum eigenvalue feature vector of $\mathbf{M}$.

2.2. Calculations of $T^{2}$ and SPE Statistic for Kernel Neighborhood Preserving Embedding. The method based on KNPE process monitoring can reduce $t$, the false alarm rate and missed alarm rate, and improve the accuracy by separating the data space into global public space and the local special subspace.

We project the matrix from the high dimension space to low dimension space as $\boldsymbol{\Phi}\left(\mathbf{X}_{g}\right)=\mathbf{G}^{T} \boldsymbol{\Phi}(\mathbf{X})$. According to (4), we can obtain

$$
\begin{aligned}
& \quad-2 \lambda \mathbf{G}=2 \boldsymbol{\Phi}(\mathbf{X}) \mathbf{M} \boldsymbol{\Phi}^{T}(\mathbf{X}) \mathbf{G}-2 \lambda \mathbf{G}=\mathbf{0}_{v \times d} \\
& \frac{\partial L}{\partial \lambda}=\mathbf{G}^{T} \mathbf{G}-\mathbf{I}_{d}=\mathbf{0}_{d \times d} .
\end{aligned}
$$

Setting $\mathbf{G}=\boldsymbol{\Phi}(\mathbf{X}) \mathbf{S}$, coefficient matrix is $\mathbf{S}=\left[\mathbf{s}_{1}, \ldots, \mathbf{s}_{d}\right] \epsilon$ $\mathbf{R}^{n}$. Equation (9) can be transformed to 


$$
\begin{gathered}
\boldsymbol{\Phi}(\mathbf{X}) \mathbf{M} \Phi^{T}(\mathbf{X}) \Phi(\mathbf{X}) \mathbf{S}=\lambda \Phi(\mathbf{X}) \mathbf{S} \\
\boldsymbol{\Phi}^{T}(\mathbf{X}) \boldsymbol{\Phi}(\mathbf{X}) \mathbf{M} \boldsymbol{\Phi}^{T}(\mathbf{X}) \boldsymbol{\Phi}(\mathbf{X}) \mathbf{S}=\lambda \boldsymbol{\Phi}^{T}(\mathbf{X}) \boldsymbol{\Phi}(\mathbf{X}) \mathbf{S} \\
\mathbf{K M K S}=\lambda \mathbf{K S} .
\end{gathered}
$$

Perform eigenvalue decomposition of matrix MK. And then the projection mapping matrix $\mathbf{S}$ from high dimension space to low dimension space can be gotten by reconstructing eigenvector referring to the $d$ smallest eigenvalue.

Based on the subspace KNPE separation modeling process is as follows:

$$
\begin{aligned}
\boldsymbol{\Phi}^{T}(\mathbf{X}) & =\boldsymbol{\Phi}^{T}\left(\mathrm{X}_{g}\right) \mathbf{G}^{T}+\boldsymbol{\Phi}\left(\mathrm{X}_{l}\right) \\
& =\boldsymbol{\Phi}^{T}\left(\mathrm{X}_{g}\right) \mathbf{G}^{T}+\left[\boldsymbol{\Phi}\left(\mathrm{X}_{l, 1}\right), \ldots, \boldsymbol{\Phi}\left(\mathrm{X}_{l, \mathrm{C}}\right)\right]
\end{aligned}
$$

where $\boldsymbol{\Phi}\left(\mathbf{X}_{g}\right)$ is global public subspace, $\boldsymbol{\Phi}\left(\mathbf{X}_{l}\right)$ is each local special subspace, $\boldsymbol{\Phi}\left(\mathbf{X}_{l, i}\right) \in \mathbf{R}^{v}, i=1, \ldots, C$, and $C$ is the number of modes in the multiple mode process.

For the new sample data $\boldsymbol{\Phi}\left(\mathbf{X}_{\text {new }}\right)$, the global public subspace Hotelling $T^{2}$ statistics and SPE statistics of each local special subspace are as follows:

$$
\begin{aligned}
& \mathbf{L}_{\text {new }}=\mathbf{G}^{T} \boldsymbol{\Phi}\left(\mathbf{X}_{\text {new }}\right)=\mathbf{S}^{T} \boldsymbol{\Phi}^{T}(\mathbf{X}) \boldsymbol{\Phi}\left(\mathbf{X}_{\text {new }}\right) \\
& =\mathbf{S}^{T} \mathbf{K}^{T}\left(\mathbf{X}_{\text {new }}, \mathbf{X}\right) \\
& T_{g}^{2}=\mathbf{L}_{\text {new }}^{T} \Lambda^{-1} \mathbf{L}_{\text {new }} \\
& \mathrm{SPE}=\left\|\widetilde{\boldsymbol{\Phi}}\left(\mathbf{X}_{\text {new }}\right)\right\|^{2}=\left\|\left(\boldsymbol{\Phi}^{T}\left(\mathbf{X}_{\text {new }}\right)-\mathbf{L}_{\text {new }}^{T} \mathbf{G}^{T}\right)\right\|^{2} \\
& =\left\|\left(\boldsymbol{\Phi}^{T}\left(\mathbf{X}_{\text {new }}\right)-\boldsymbol{\Phi}^{T}\left(\mathbf{X}_{\text {new }}\right) \boldsymbol{\Phi}(\mathbf{X}) \mathbf{S S}^{T} \boldsymbol{\Phi}^{T}(\mathbf{X})\right)\right\|^{2} \\
& =\left\|\boldsymbol{\Phi}^{T}\left(\mathbf{X}_{\text {new }}\right)\left(\mathbf{I}-\boldsymbol{\Phi}(\mathbf{X}) \mathbf{S S}^{T} \boldsymbol{\Phi}^{T}(\mathbf{X})\right)\right\|^{2} \\
& =\left\|\left(\mathbf{I}-\boldsymbol{\Phi}(\mathbf{X}) \mathbf{S S}^{T} \boldsymbol{\Phi}^{T}(\mathbf{X})\right) \boldsymbol{\Phi}\left(\mathbf{X}_{\text {new }}\right)\right\|^{2} \\
& =\left(\left(\mathbf{I}-\boldsymbol{\Phi}(\mathbf{X}) \mathbf{S S}^{T} \boldsymbol{\Phi}^{T}(\mathbf{X})\right) \boldsymbol{\Phi}\left(\mathbf{X}_{\text {new }}\right)\right)^{T} \\
& \cdot\left(\left(\mathbf{I}-\boldsymbol{\Phi}(\mathbf{X}) \mathbf{S S}^{T} \boldsymbol{\Phi}^{T}(\mathbf{X})\right) \boldsymbol{\Phi}\left(\mathbf{X}_{\text {new }}\right)\right)=\boldsymbol{\Phi}^{T}\left(\mathbf{X}_{\text {new }}\right) \\
& \cdot\left(\mathbf{I}-\boldsymbol{\Phi}(\mathbf{X}) \mathbf{S S}^{T} \boldsymbol{\Phi}^{T}(\mathbf{X})\right)(\mathbf{I} \\
& \text { - } \left.\boldsymbol{\Phi}(\mathrm{X}) \boldsymbol{S S}^{T} \boldsymbol{\Phi}^{T}(\mathrm{X})\right) \boldsymbol{\Phi}\left(\mathrm{X}_{\text {new }}\right)=\boldsymbol{\Phi}^{T}\left(\mathrm{X}_{\text {new }}\right)(\mathbf{I} \\
& -2 \Phi(\mathbf{X}) \mathbf{S S}^{T} \boldsymbol{\Phi}^{T}(\mathbf{X}) \\
& \left.+\boldsymbol{\Phi}(\mathrm{X}) \mathbf{S S}^{T} \boldsymbol{\Phi}^{T}(\mathrm{X}) \boldsymbol{\Phi}(\mathrm{X}) \mathbf{S S}^{T} \boldsymbol{\Phi}^{T}(\mathrm{X})\right) \boldsymbol{\Phi}\left(\mathrm{X}_{\mathrm{new}}\right) \\
& =\Phi^{T}\left(\mathrm{X}_{\text {new }}\right) \Phi\left(\mathrm{X}_{\text {new }}\right)-2 \Phi^{T}\left(\mathrm{X}_{\text {new }}\right) \boldsymbol{\Phi}(\mathbf{X}) \\
& \cdot \operatorname{SS}^{T} \boldsymbol{\Phi}^{T}(\mathrm{X}) \boldsymbol{\Phi}\left(\mathrm{X}_{\mathrm{new}}\right)+\boldsymbol{\Phi}^{T}\left(\mathrm{X}_{\text {new }}\right) \boldsymbol{\Phi}(\mathrm{X}) \\
& \cdot \mathbf{S S}^{T} \boldsymbol{\Phi}^{T}(\mathbf{X}) \boldsymbol{\Phi}(\mathrm{X}) \mathbf{S S}^{T} \boldsymbol{\Phi}^{T}(\mathbf{X}) \boldsymbol{\Phi}\left(\mathrm{X}_{\text {new }}\right) \\
& =\mathbf{K}\left(\mathbf{X}_{\text {new }}, \mathbf{X}_{\text {new }}\right)-2 \mathbf{K}\left(\mathbf{X}_{\text {new }}, \mathbf{X}\right) \mathbf{S S}^{T} \mathbf{K}^{T}\left(\mathbf{X}_{\text {new }}, \mathbf{X}\right) \\
& +\mathbf{K}\left(\mathbf{X}_{\text {new }}, \mathbf{X}\right) \mathbf{S S}^{T} \mathbf{K S S}^{T} \mathbf{K}^{T}\left(\mathbf{X}_{\text {new }}, \mathbf{X}\right),
\end{aligned}
$$

where $\Lambda=\boldsymbol{\Phi}\left(\mathbf{X}_{g}\right) \Phi^{T}\left(\mathbf{X}_{g}\right) / n-1$ is covariance matrix of global public subspace on the training set.

\section{Quality-Relevant Kernel Neighborhood Preserving Embedding Method}

3.1. Theory of Quality-Relevant Kernel Neighborhood Preserving Embedding. KNPE method can deal with the nonlinear problem better, but it did not consider the change of process quality variables which industrial process is most concerned with. That is one of the reasons why false alarm and missed alarm happen frequently. The covariance information represents relationship of global public subspace and quality variables. In multimodal subspace separation, we make global public space $\boldsymbol{\Phi}\left(\mathbf{X}_{g}\right)=\left[\boldsymbol{\Phi}\left(\mathbf{x}_{g, 1}\right), \ldots, \boldsymbol{\Phi}\left(\mathbf{x}_{g, d}\right)\right] \in \mathbf{R}^{n}$ to keep the local structural information of high dimension space of input data and at the same time make the covariance information of score matrix of output quality variable $\mathbf{Y}=\left[\mathbf{y}_{1}, \ldots, \mathbf{y}_{n}\right] \in \mathbf{R}^{m}$ after standardization largest. $\mathbf{T}=\left[\mathbf{t}_{1}, \ldots, \mathbf{t}_{d}\right] \in \mathbf{R}^{n}$ is score matrix of input data variable $\boldsymbol{\Phi}^{T}(\mathbf{X})$ of each mode, where $\mathbf{T}=\boldsymbol{\Phi}^{T}(\mathbf{X}) \mathbf{R}$ and $\mathbf{R}=\left[\mathbf{r}_{1}, \ldots, \mathbf{r}_{d}\right] \in \mathbf{R}^{v}$ is the load matrix of $\boldsymbol{\Phi}^{T}(\mathbf{X}) . \mathbf{U}=\left[\mathbf{u}_{1}, \ldots, \mathbf{u}_{d}\right] \in \mathbf{R}^{n}$ is score matrix of each model output variable. Namely, $\mathbf{U}=\mathbf{Y}^{T} \mathbf{C} . \mathbf{C}=\left[\mathbf{c}_{1}, \ldots, \mathbf{c}_{d}\right] \in \mathbf{R}^{m}$ is load matrix of $\mathbf{Y}^{T} . n$ is the total length of various models. The relationship of global public subspace and quality variable information is fully extracted. The extraction of the global public space will meet requirements of complex industrial process and improve the accuracy of the multimode process monitoring. The model using QKNPE method to extract the global public subspace is as follows:

$$
\begin{array}{ll}
\min \quad J & \\
= & \operatorname{tr}\left(\boldsymbol{\Phi}\left(\mathbf{x}_{g, i}\right) \mathbf{M} \boldsymbol{\Phi}^{T}\left(\mathbf{x}_{g, i}\right)\right) \\
& -\boldsymbol{\Phi}\left(\mathbf{x}_{g, i}\right) \boldsymbol{\Phi}^{T}(\mathbf{X}) \mathbf{r}_{i}-\boldsymbol{\Phi}\left(\mathbf{x}_{g, i}\right) \mathbf{Y}^{T} \mathbf{c}_{i} \\
\text { s.t. } \quad & \boldsymbol{\Phi}^{T}\left(\mathbf{x}_{g, i}\right) \boldsymbol{\Phi}\left(\mathbf{x}_{g, i}\right)=\mathbf{I}_{n} \\
& \mathbf{r}_{i}^{T} \mathbf{r}_{i}=1 \\
& \mathbf{c}_{i}^{T} \mathbf{c}_{i}=1,
\end{array}
$$

where $\min \operatorname{tr}\left(\boldsymbol{\Phi}\left(\mathbf{x}_{g, i}\right) \mathbf{M} \boldsymbol{\Phi}^{T}\left(\mathbf{x}_{g, i}\right)\right)$ corresponds to (4) and $\min -\boldsymbol{\Phi}\left(\mathbf{x}_{g, i}\right) \boldsymbol{\Phi}^{T}(\mathbf{X}) \mathbf{r}_{i}-\boldsymbol{\Phi}\left(\mathbf{x}_{g, i}\right) \mathbf{Y}^{T} \mathbf{c}_{i}$ means maximizing the projection of global public data along the score directions of $\boldsymbol{\Phi}^{T}(\mathbf{X})$ and $\mathbf{Y}^{T}$. Thus, we can make the point $\boldsymbol{\Phi}\left(\mathbf{x}_{g, i}\right)$ in the global public subspace establish the relations with quality variables. $\Phi\left(\mathbf{x}_{g, i}\right)$ is obtained by using the Lagrange multiplier method. The Lagrange function is as follows:

$$
\begin{aligned}
L= & \operatorname{tr}\left(\boldsymbol{\Phi}\left(\mathbf{x}_{g, i}\right) \mathbf{M} \boldsymbol{\Phi}^{T}\left(\mathbf{x}_{g, i}\right)\right)-\boldsymbol{\Phi}\left(\mathbf{x}_{g, i}\right) \boldsymbol{\Phi}^{T}(\mathbf{X}) \mathbf{r}_{i} \\
& -\boldsymbol{\Phi}\left(\mathbf{x}_{g, i}\right) \mathbf{Y}^{T} \mathbf{c}_{i}-\lambda_{1}\left(\boldsymbol{\Phi}^{T}\left(\mathbf{x}_{g, i}\right) \boldsymbol{\Phi}\left(\mathbf{x}_{g, i}\right)-\mathbf{I}_{n}\right) \\
& -\lambda_{2}\left(\mathbf{r}_{i}^{T} \mathbf{r}_{i}-1\right)-\lambda_{3}\left(\mathbf{c}_{i}^{T} \mathbf{c}_{i}-1\right) .
\end{aligned}
$$


Setting the partial derivative of Lagrange function for $\Phi\left(\mathbf{x}_{g, i}\right), \mathbf{r}_{i}, \mathbf{c}_{i}$, and $\lambda_{i}$ as 0 ,

$$
\begin{aligned}
\frac{\partial L}{\partial \Phi\left(\mathbf{x}_{g, i}\right)}= & \boldsymbol{\Phi}\left(\mathbf{x}_{g, i}\right) \mathbf{M}+\boldsymbol{\Phi}\left(\mathbf{x}_{g, i}\right) \mathbf{M}^{T}-\mathbf{r}_{i}^{T} \boldsymbol{\Phi}(\mathbf{X}) \\
& -\mathbf{c}_{i}^{T} \mathbf{Y}-2 \lambda_{1} \boldsymbol{\Phi}\left(\mathbf{x}_{g, i}\right) \\
= & 2 \boldsymbol{\Phi}\left(\mathbf{x}_{g, i}\right) \mathbf{M}-\mathbf{r}_{i}^{T} \boldsymbol{\Phi}(\mathbf{X})-\mathbf{c}_{i}^{T} \mathbf{Y} \\
& -2 \lambda_{1} \boldsymbol{\Phi}\left(\mathbf{x}_{g, i}\right)=\mathbf{0}_{1 \times n} \\
\frac{\partial L}{\partial \mathbf{r}_{i}}= & -\boldsymbol{\Phi}(\mathbf{X}) \boldsymbol{\Phi}^{T}\left(\mathbf{x}_{g, i}\right)-2 \lambda_{2} \mathbf{r}_{i}=\mathbf{0}_{v \times 1} \\
\frac{\partial L}{\partial \mathbf{c}_{i}}= & -\mathbf{Y} \boldsymbol{\Phi}^{T}\left(\mathbf{x}_{g, i}\right)-2 \lambda_{3} \mathbf{c}_{i}=\mathbf{0}_{m \times 1} \\
\frac{\partial L}{\partial \lambda_{1}}= & \boldsymbol{\Phi}\left(\mathbf{x}_{g, i}\right)^{T} \boldsymbol{\Phi}\left(\mathbf{x}_{g, i}\right)-\mathbf{I}_{n}=\mathbf{0}_{n} \\
\frac{\partial L}{\partial \lambda_{2}}= & \mathbf{r}_{i}^{T} \mathbf{r}_{i}-1=0 \\
\frac{\partial L}{\partial \lambda_{3}}= & \mathbf{c}_{i}^{T} \mathbf{c}_{i}-1=0 .
\end{aligned}
$$

It is available by deriving

$$
\begin{aligned}
& 2 \mathbf{M} \boldsymbol{\Phi}^{T}\left(\mathbf{x}_{g, i}\right)-\boldsymbol{\Phi}^{T}(\mathbf{X}) \mathbf{r}_{i}-\mathbf{Y}^{T} \mathbf{c}_{i}=2 \lambda_{1} \boldsymbol{\Phi}^{T}\left(\mathbf{x}_{g, i}\right) \\
& \boldsymbol{\Phi}^{T}(\mathbf{X}) \mathbf{r}_{i}=-\frac{1}{2 \lambda_{2}} \boldsymbol{\Phi}^{T}(\mathbf{X}) \boldsymbol{\Phi}(\mathbf{X}) \boldsymbol{\Phi}^{T}\left(\mathbf{x}_{g, i}\right) \\
& \mathbf{Y}^{T} \mathbf{c}_{i}=-\frac{1}{2 \lambda_{3}} \mathbf{Y}^{T} \mathbf{Y} \boldsymbol{\Phi}^{T}\left(\mathbf{x}_{g, i}\right) .
\end{aligned}
$$
form:

The only least solution $\boldsymbol{\Phi}\left(\mathbf{x}_{g, i}\right)$ will meet the following

$$
\begin{aligned}
& \left(2 \mathbf{M}+\frac{\boldsymbol{\Phi}^{T}(\mathbf{X}) \boldsymbol{\Phi}(\mathbf{X})}{\left(2 \lambda_{2}\right)}+\frac{\mathbf{Y}_{j}^{T} \mathbf{Y}_{j}}{\left(2 \lambda_{3}\right)}\right) \boldsymbol{\Phi}^{T}\left(\mathbf{x}_{g, i}\right) \\
& =2 \lambda_{1} \boldsymbol{\Phi}^{T}\left(\mathbf{x}_{g, i}\right) .
\end{aligned}
$$

So the feature decomposition of matrix $\left(2 \mathbf{M}+\mathbf{K} /\left(2 \lambda_{2}\right)+\right.$ $\left.\mathbf{Y}_{j}^{T} \mathbf{Y}_{j} /\left(2 \lambda_{3}\right)\right)$ is made. The eigenvectors which the $d$ smallest eigenvalue corresponds to are the best refactoring point matrix $\boldsymbol{\Phi}\left(\mathbf{X}_{g}\right)$. The load vector of each model input data and output quality variables is

$$
\begin{aligned}
& \mathbf{r}_{i}=-\frac{\boldsymbol{\Phi}(\mathbf{X}) \boldsymbol{\Phi}^{T}\left(\mathbf{x}_{g, i}\right)}{2 \lambda_{2}} \\
& \mathbf{c}_{i}=-\frac{\mathbf{Y} \boldsymbol{\Phi}^{T}\left(\mathbf{x}_{g, i}\right)}{2 \lambda_{3}} .
\end{aligned}
$$

3.2. Process Monitoring Based on Quality-Relevant Kernel Neighborhood Preserving Embedding. According to (12), we set $\boldsymbol{\Phi}\left(\mathbf{x}_{g, i}\right)=\mathbf{G}_{i}^{T} \boldsymbol{\Phi}(\mathbf{X})$ and

$$
\begin{aligned}
\min \mathbf{e}\left(\mathbf{G}_{i}\right) & \\
= & \operatorname{trace}\left(\mathbf{G}_{i}^{T} \boldsymbol{\Phi}(\mathbf{X}) \mathbf{M} \boldsymbol{\Phi}^{T}(\mathbf{X}) \mathbf{G}_{i}\right) \\
& +\frac{\mathbf{G}_{i}^{T} \boldsymbol{\Phi}(\mathbf{X}) \boldsymbol{\Phi}^{T}(\mathbf{X}) \boldsymbol{\Phi}(\mathbf{X}) \boldsymbol{\Phi}^{T}(\mathbf{X}) \mathbf{G}_{i}}{2 \lambda_{2}} \\
& +\frac{\mathbf{G}_{i}^{T} \boldsymbol{\Phi}(\mathbf{X}) \mathbf{Y}^{T} \mathbf{Y} \boldsymbol{\Phi}^{T}(\mathbf{X}) \mathbf{G}_{i}}{2 \lambda_{3}}
\end{aligned}
$$

s.t. $\mathbf{G}_{i}^{T} \mathbf{G}_{i}=1$,

where $\mathbf{G}=\left[\mathbf{G}_{1}, \mathbf{G}_{2}, \ldots, \mathbf{G}_{d}\right] \in \mathbf{R}^{v}$ is mapping matrix from high dimension space to low dimension space. The constraint condition is $\mathbf{G}_{i}^{T} \mathbf{G}_{i}=1$. And then

$$
\begin{aligned}
L= & \operatorname{trace}\left(\mathbf{G}_{i}^{T} \boldsymbol{\Phi}(\mathbf{X}) \mathbf{M} \boldsymbol{\Phi}^{T}(\mathbf{X}) \mathbf{G}_{i}\right) \\
& +\frac{\mathbf{G}_{i}^{T} \boldsymbol{\Phi}(\mathbf{X}) \boldsymbol{\Phi}^{T}(\mathbf{X}) \boldsymbol{\Phi}(\mathbf{X}) \boldsymbol{\Phi}^{T}(\mathbf{X}) \mathbf{G}_{i}}{2 \lambda_{2}} \\
& +\frac{\mathbf{G}_{i}^{T} \boldsymbol{\Phi}(\mathbf{X}) \mathbf{Y}^{T} \mathbf{Y} \boldsymbol{\Phi}^{T}(\mathbf{X}) \mathbf{G}_{i}}{2 \lambda_{3}}-\lambda\left(\mathbf{G}_{i}^{T} \mathbf{G}_{i}-1\right)
\end{aligned}
$$$$
\frac{\partial L}{\partial \mathbf{G}_{i}}=\boldsymbol{\Phi}(\mathbf{X}) \mathbf{M} \boldsymbol{\Phi}^{T}(\mathbf{X}) \mathbf{G}_{i}+\left(\boldsymbol{\Phi}(\mathbf{X}) \mathbf{M} \boldsymbol{\Phi}^{T}(\mathbf{X})\right)^{T} \mathbf{G}_{i}
$$$$
+\frac{\Phi(\mathbf{X}) \Phi^{T}(\mathbf{X}) \boldsymbol{\Phi}(\mathbf{X}) \Phi^{T}(\mathbf{X}) \mathbf{G}_{i}}{2 \lambda_{2}}
$$$$
+\frac{\left(\boldsymbol{\Phi}(\mathbf{X}) \boldsymbol{\Phi}^{T}(\mathbf{X}) \boldsymbol{\Phi}(\mathbf{X}) \boldsymbol{\Phi}^{T}(\mathbf{X})\right)^{T} \mathbf{G}_{i}}{2 \lambda_{2}}
$$$$
+\frac{\Phi(\mathbf{X}) \mathbf{Y}^{T} \mathbf{Y} \Phi^{T}(\mathbf{X}) \mathbf{G}_{i}}{2 \lambda_{3}}
$$$$
+\frac{\left(\boldsymbol{\Phi}(\mathbf{X}) \mathbf{Y}^{T} \mathbf{Y} \boldsymbol{\Phi}^{T}(\mathbf{X}) \mathbf{G}_{i}\right)^{T}}{2 \lambda_{3}}-2 \lambda \mathbf{G}_{i}
$$$$
=2 \Phi(\mathbf{X}) \mathbf{M} \boldsymbol{\Phi}^{T}(\mathbf{X}) \mathbf{G}_{i}+\frac{\boldsymbol{\Phi}(\mathbf{X}) \mathbf{K} \boldsymbol{\Phi}^{T}(\mathbf{X}) \mathbf{G}_{i}}{\lambda_{2}}
$$$$
+\frac{\boldsymbol{\Phi}(\mathbf{X}) \mathbf{Y}^{T} \mathbf{Y} \boldsymbol{\Phi}^{T}(\mathbf{X}) \mathbf{G}_{i}}{\lambda_{3}}-2 \lambda \mathbf{G}_{i}=\mathbf{0}_{v \times 1}
$$

$$
\frac{\partial L}{\partial \lambda}=\mathbf{G}_{i}^{T} \mathbf{G}_{i}-1=0 .
$$




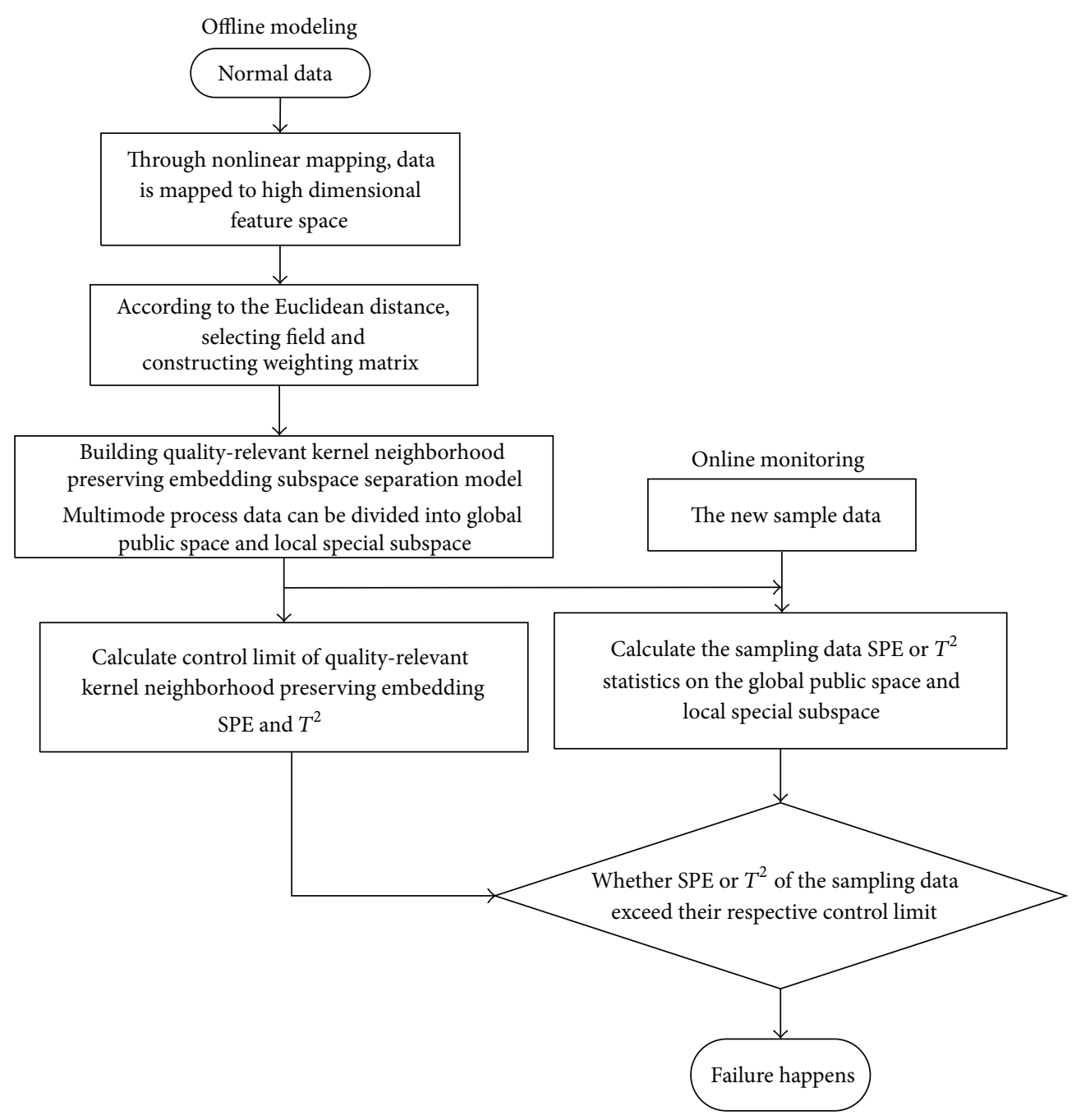

FIGURE 2: The monitoring flow of multimode processes based on QKNPE.

Let $\mathbf{G}_{i}=\boldsymbol{\Phi}(\mathbf{X}) \mathbf{S}_{i}$ and let $\mathbf{S}=\left[\mathbf{S}_{1}, \ldots, \mathbf{S}_{d}\right] \in \mathbf{R}^{n} ;(20)$ can be transformed as

$$
\begin{aligned}
& 2 \Phi(\mathbf{X}) \mathbf{M} \Phi^{T}(\mathbf{X}) \boldsymbol{\Phi}(\mathbf{X}) \mathbf{S}_{i}+\frac{\Phi(\mathbf{X}) \mathbf{K} \Phi^{T}(\mathbf{X}) \boldsymbol{\Phi}(\mathbf{X}) \mathbf{S}_{i}}{\lambda_{2}} \\
& +\frac{\Phi(\mathbf{X}) \mathbf{Y}^{T} \mathbf{Y} \Phi^{T}(\mathbf{X}) \Phi(\mathbf{X}) \mathbf{S}_{i}}{\lambda_{3}}=2 \lambda \Phi(\mathbf{X}) \mathbf{S}_{i} \\
& 2 \Phi^{T}(\mathbf{X}) \boldsymbol{\Phi}(\mathbf{X}) \mathbf{M} \Phi^{T}(\mathbf{X}) \boldsymbol{\Phi}(\mathbf{X}) \mathbf{S}_{i} \\
& +\frac{\Phi^{T}(\mathbf{X}) \Phi(\mathbf{X}) \mathbf{K} \Phi^{T}(\mathbf{X}) \Phi(\mathbf{X}) \mathbf{S}_{i}}{\lambda_{2}} \\
& +\frac{\boldsymbol{\Phi}^{T}(\mathbf{X}) \boldsymbol{\Phi}(\mathbf{X}) \mathbf{Y}^{T} \mathbf{Y} \boldsymbol{\Phi}^{T}(\mathbf{X}) \boldsymbol{\Phi}(\mathbf{X}) \mathbf{S}_{i}}{\lambda_{3}} \\
& =2 \lambda \Phi^{T}(\mathbf{X}) \boldsymbol{\Phi}(\mathbf{X}) \mathbf{S}_{i} \\
& 2 \mathbf{K M K S}_{i}+\frac{\mathbf{K K K S}_{i}}{\lambda_{2}}+\frac{\mathbf{K Y}^{T} \mathbf{Y K S}_{i}}{\lambda_{3}}=2 \lambda \mathbf{K S}_{i} .
\end{aligned}
$$

The minimal solution of $\mathbf{S}_{i}$ meets the following form:

$$
\left(2 \mathbf{M K}+\frac{\mathbf{K K}}{\lambda_{2}}+\frac{\mathbf{Y}^{T} \mathbf{Y K}}{\lambda_{3}}\right) \mathbf{S}_{i}=2 \lambda \mathbf{S}_{i}
$$

So the feature decomposition for matrix $\left(2 \mathbf{M K}+\mathbf{K K} / \lambda_{2}+\right.$ $\left.\mathbf{Y}^{T} \mathbf{Y K} / \lambda_{3}\right)$ is made. The eigenvector corresponding to the $d$ smallest eigenvalue is projection mapping matrix $\mathbf{S}_{i}$ from high dimension space to low dimension space.

Thus, the following equation can be gotten:

$$
\begin{aligned}
\boldsymbol{\Phi}^{T}(\mathbf{X}) & =\boldsymbol{\Phi}^{T}\left(\mathbf{X}_{g}\right) \mathbf{G}^{T}+\boldsymbol{\Phi}\left(\mathbf{X}_{l}\right) \\
& =\boldsymbol{\Phi}^{T}\left(\mathbf{X}_{g}\right) \mathbf{G}^{T}+\left[\boldsymbol{\Phi}\left(\mathbf{X}_{l, 1}\right), \ldots, \boldsymbol{\Phi}\left(\mathbf{X}_{l, C}\right)\right]
\end{aligned}
$$

where $\boldsymbol{\Phi}\left(\mathbf{X}_{l}\right)$ are each local special subspace and $\Phi\left(\mathbf{X}_{l, i}\right) \in \mathbf{R}^{v}$, $i=1, \ldots, C$.

The flow chart of the whole multimode process monitoring method is shown in Figure 2. 
TABLE 1: The definition of different modes in EFMF.

\begin{tabular}{lcc}
\hline Mode & Raw material & $\begin{array}{c}\text { Whether to add } \\
\text { raw material }\end{array}$ \\
\hline 1 & Magnesite block & YES \\
2 & Magnesite block & NO \\
3 & Mixture & YES \\
4 & Mixture & NO \\
5 & Magnesite powder & YES \\
6 & Magnesite powder & NO \\
\hline
\end{tabular}

\section{The Experimental Results}

The EFMF is one type of the main equipment for the production of magnesia, and it belongs to mine heat electric arc furnace. Arc is heat source. It can well smelt magnesia by concentrating heat. Overall equipment of fused magnesia furnace generally includes transformer, circuit short net, electrode lifting gear, electrode, and furnace. Furnace shell is commonly rounded and slightly tapered. For the convenience of easing shell molten lead, the ring is welded in a furnace shell wall. The mobile car is equipped below furnace, so that clinker after complete melting can be moved to fixed station and cooled [20].

The EFMF produces high temperature to complete melting process by introducing large current. The temperature of area near the electrode is high. The temperature of area far away from the electrode is low. Once the temperature of the area around electrode is too high, it is easy to cause security incident. However, once the temperature of area far away from the electrode is too low, lots of material will be wasted. It will seriously affect the product yield and quality. It is required to timely detect abnormal and failure in the process: therefore, the process monitoring for working process of fused magnesia furnace is very necessary and meaningful.

The difference of magnesite stone raw materials and the difference of charging operation are corresponding to change of characteristics of the magnesium furnace smelting process and process data has strong nonlinear characteristic. This paper selects the process data obtained through the two conditions with and without charging smelting process of the massive magnesite, mixed massive magnesite, and powder magnesite of smelting process as the six different operation modes to model. For simplifying process dividing the model, this section assumes that each model is isometric. The definition of different modes in EFMF is shown in Table 1. We use process monitoring method based on the proposed QKNPE subspace separation to monitor the work process. To show good effectiveness of the proposed method, LLE and KPLS are used for comparison. According to the comparing results, we can verify the efficiency and accuracy of the proposed QKNPE subspace separation method for monitoring the multimodal process.

Firstly, we select sampling data from six modes of EFMF for offline modeling, and all of these sampling data are obtained under normal working condition. Each group of data contains three phase current values and three key variables. The modeling data set contains 1500 samples. For validating the monitoring performance of the proposed method for multimode process, we use 6 groups which contain 1500 samples data with fault. Faults 1, 2, and 3 happen from 200th, 400th, and 700th samples, respectively. The reason is excessive heating. Faults 4, 5, and 6, which are caused by actuator stuck fault, start from 900th, 1200th, and 1400th samples, respectively.

Using multimodal process modeling and monitoring methods based on LLE, KPLS, and QKNPE subspace separation for fault 1 to fault $3, T^{2}$ and SPE statistics are shown in the diagrams in Figures 3-5. According to the results, it can be found that the local special subspace of LLE and KPLS has high nonresponse rates and low accuracy compared with QKNPE. Global public space monitoring of KPLS and QKNPE and local special subspace monitoring have high accuracy and low nonresponse rates, while QKNPE has lower rate of false positives. For faults $4-6, T^{2}$ and SPE are shown in Figures 6-8. We can find that the local special subspace of LLE monitoring for faults 4-6 has high nonresponse rates and low accuracy and the global public subspace monitoring, global public space monitoring of KPLS and QKNPE, and local special subspace monitoring have high accuracy and low nonresponse rates, while QKNPE has lower rate of false positives.

In fused magnesia furnace multimodal process, the monitoring accuracy, the rate of false positives, and nonresponse rates of six faults based on LLE, KPLS, and QKNPE subspace separation method are shown in Table 2. The experimental results show the process monitoring method based on QKNPE has very good monitoring effectiveness.

\section{Conclusion}

In this paper, the method based on QKNPE is proposed and applied to multimodal process monitoring. This method can solve the problem of nonlinear multimodal process monitoring. KPLS method can solve the problems that manifold method cannot directly be applied to nonlinear multimodal process monitoring and KPLS changes did not explain ability of quality variables. In monitoring fused magnesia furnace smelting process, QKNPE method can use subspace separation method and considering the advantages of the relationship between input variable and output variable quality, it can improve the monitoring accuracy, can reduce false positives and omission, and can be effective to detect the fault during the process of multimode.

\section{Conflict of Interests}

The authors declare that there is no conflict of interests regarding the publication of this paper. 

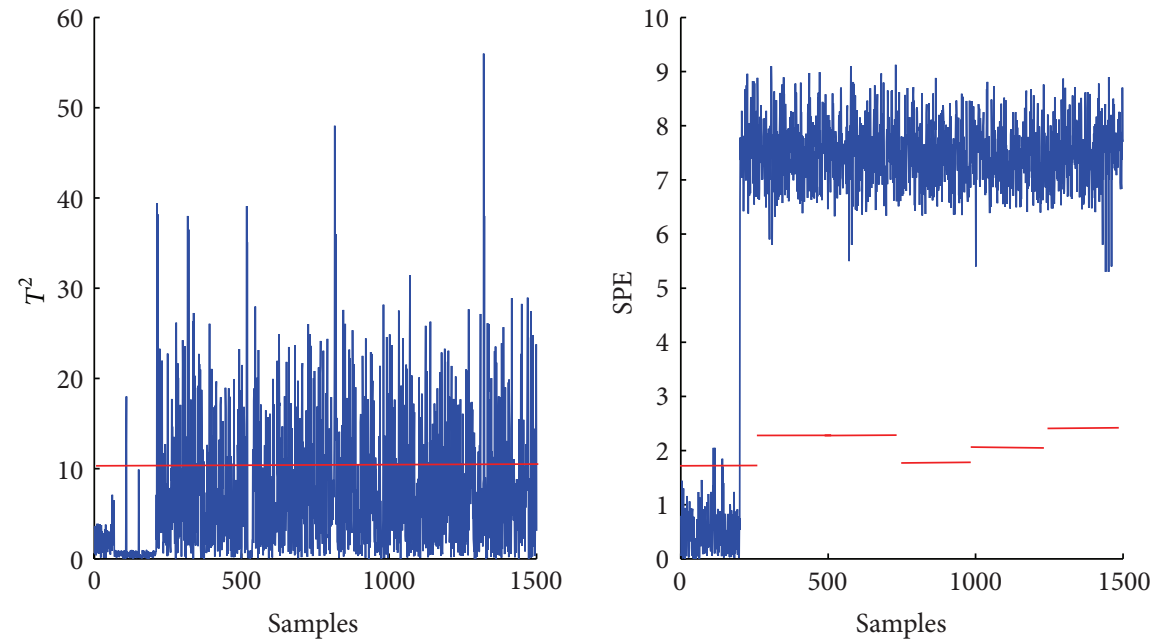

(a) Monitoring based on LLE for fault 1
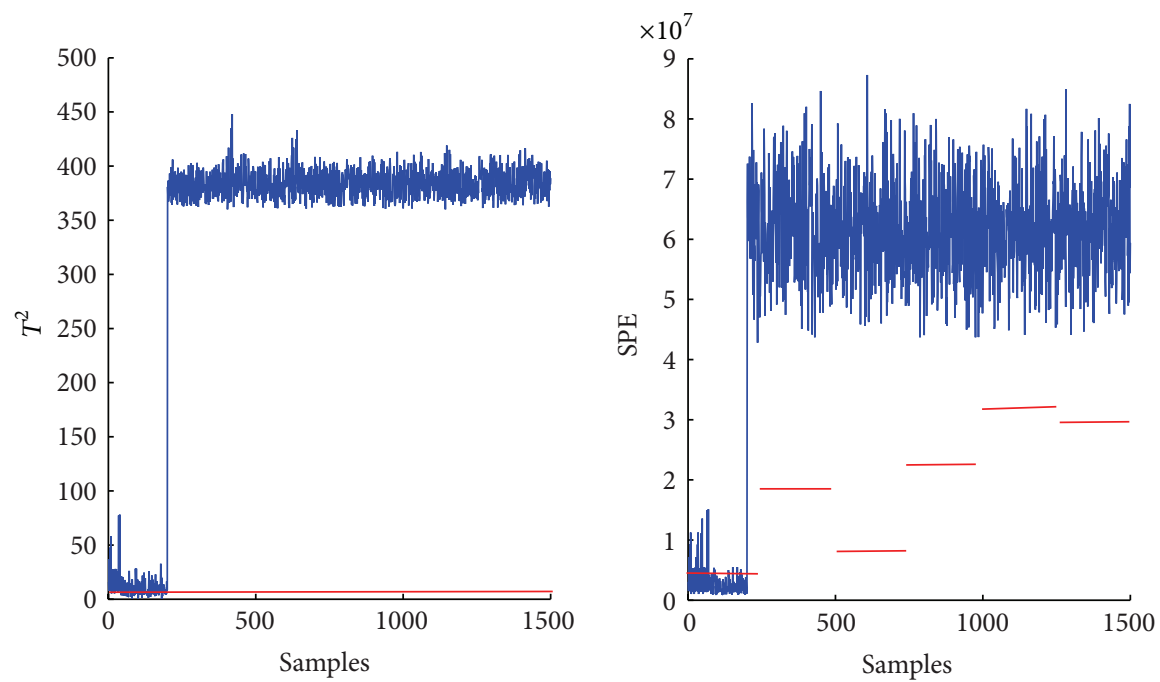

(b) Monitoring based on KPLS for fault 1
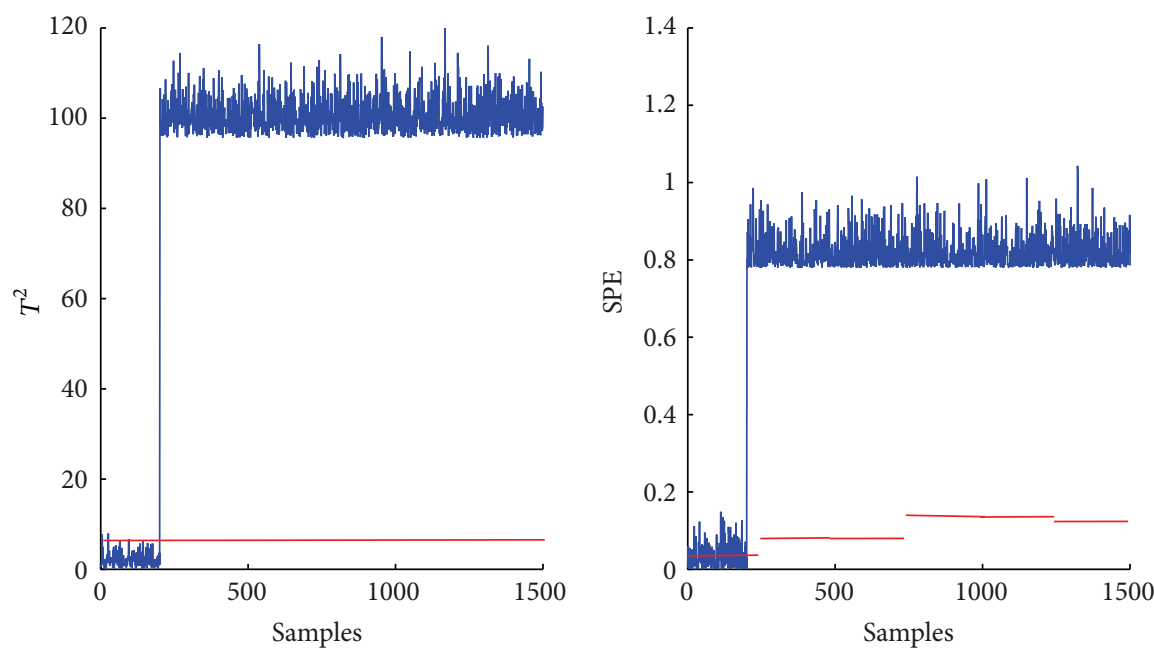

(c) Monitoring based on QKNPE for fault 1

FIgURE 3: Monitoring results for fault 1. 

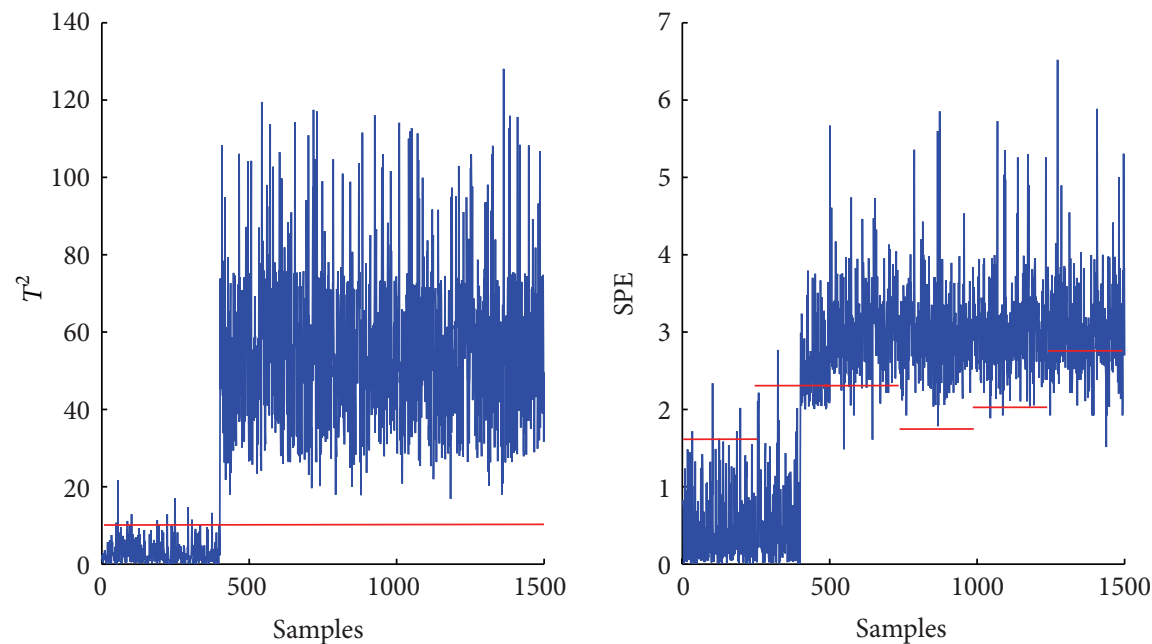

(a) Monitoring based on LLE for fault 2
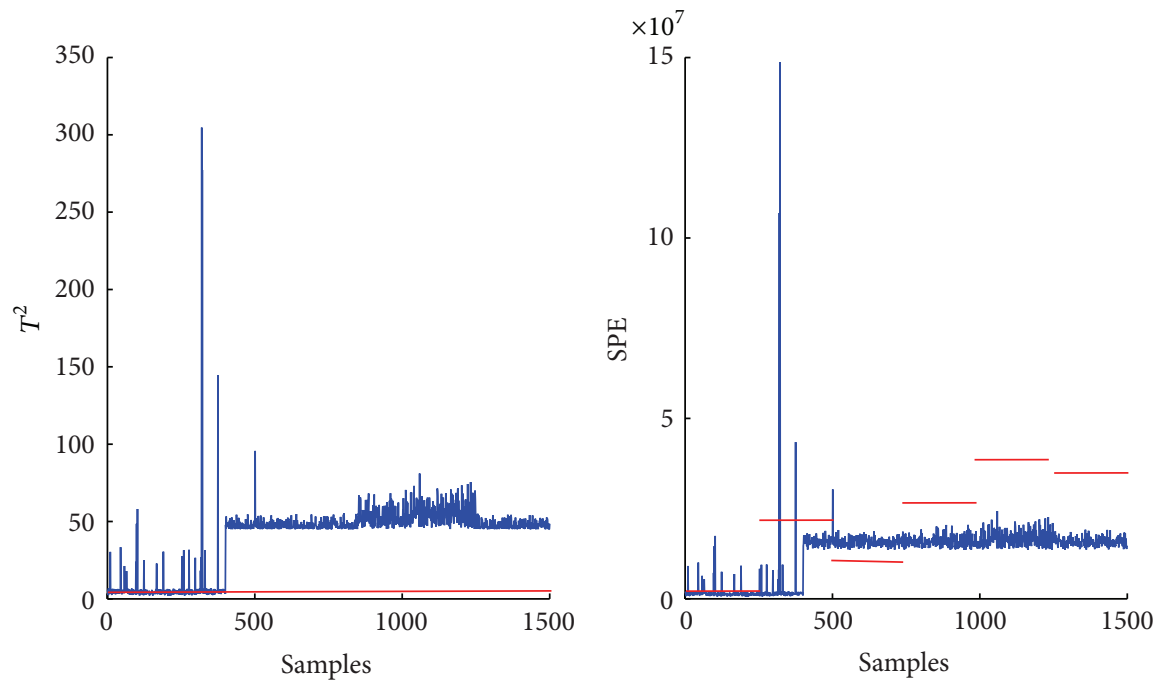

(b) Monitoring based on KPLS for fault 2
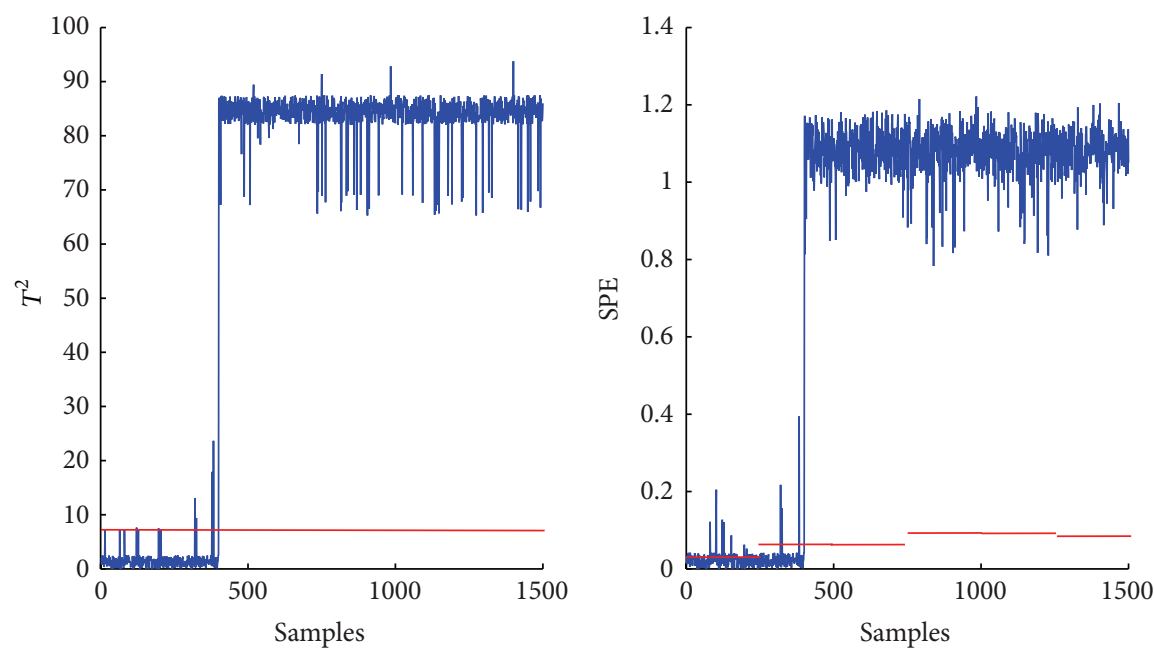

(c) Monitoring based on QKNPE for fault 2

Figure 4: Monitoring results for fault 2. 

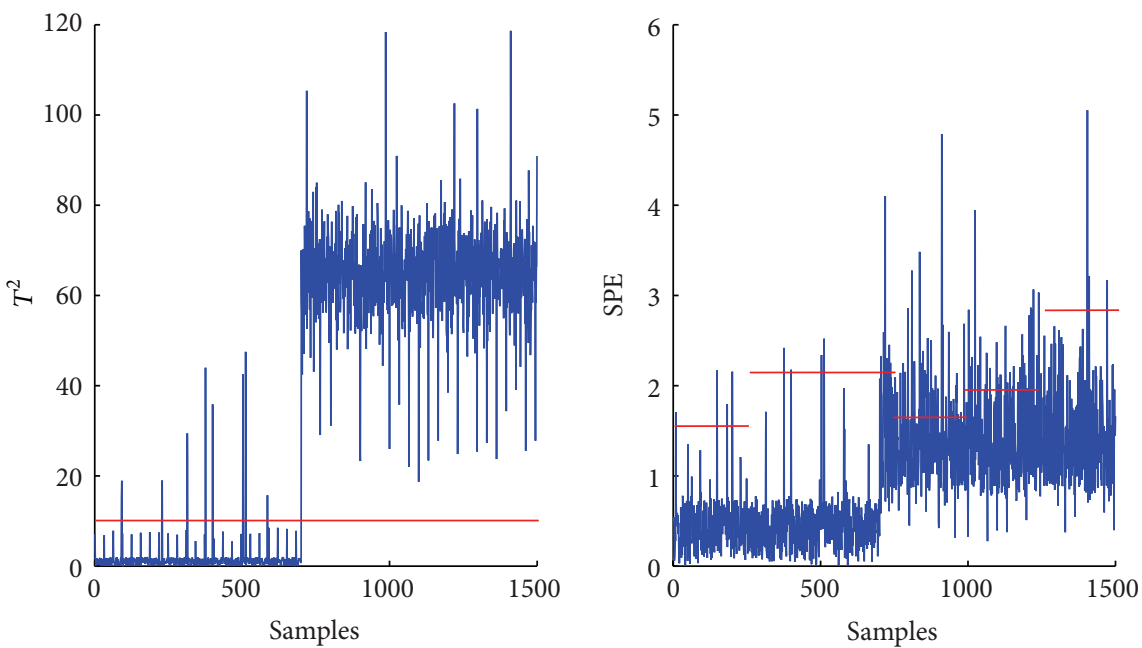

(a) Monitoring based on LLE for fault 3
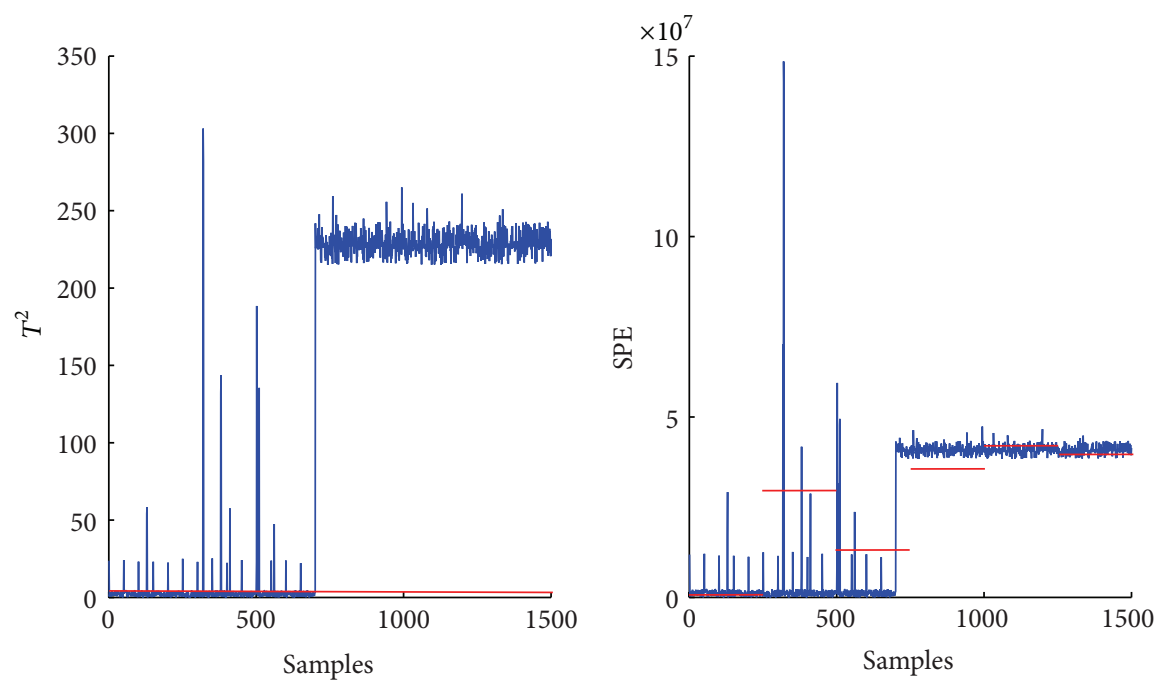

(b) Monitoring based on KPLS for fault 3
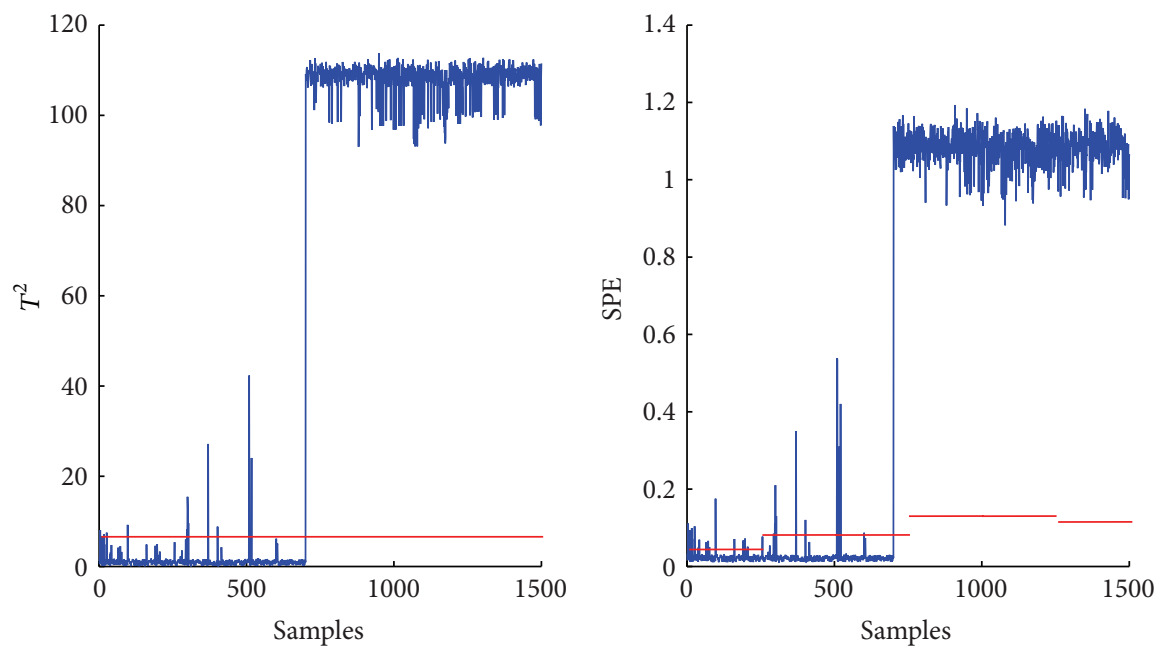

(c) Monitoring based on QKNPE for fault 3

Figure 5: Monitoring results for fault 3. 

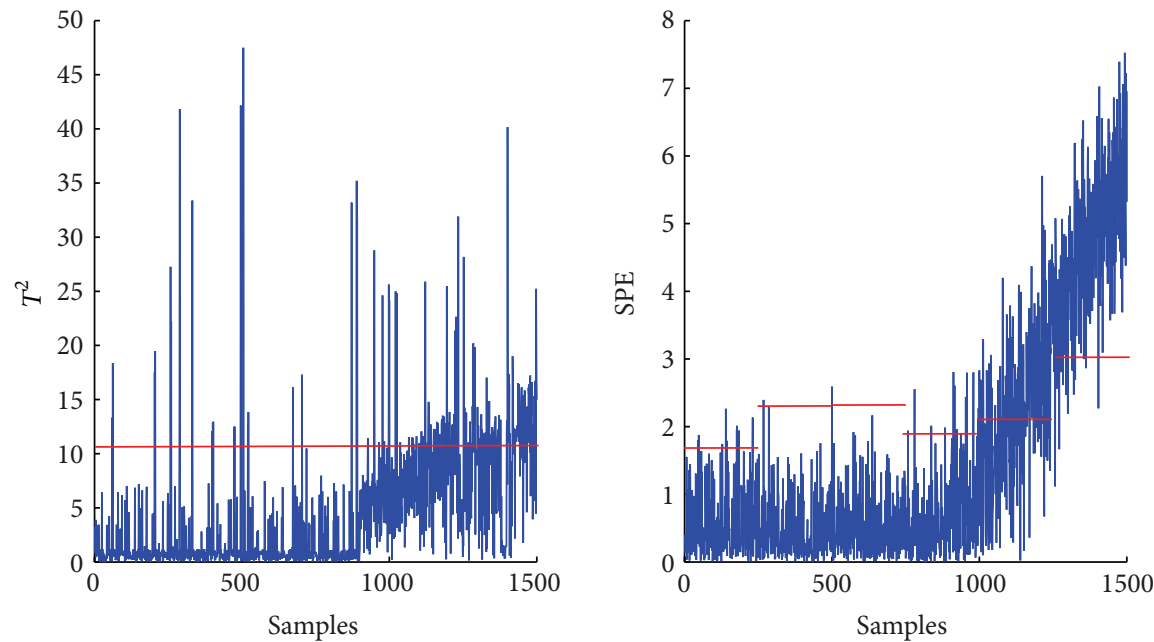

(a) Monitoring based on LLE for fault 4
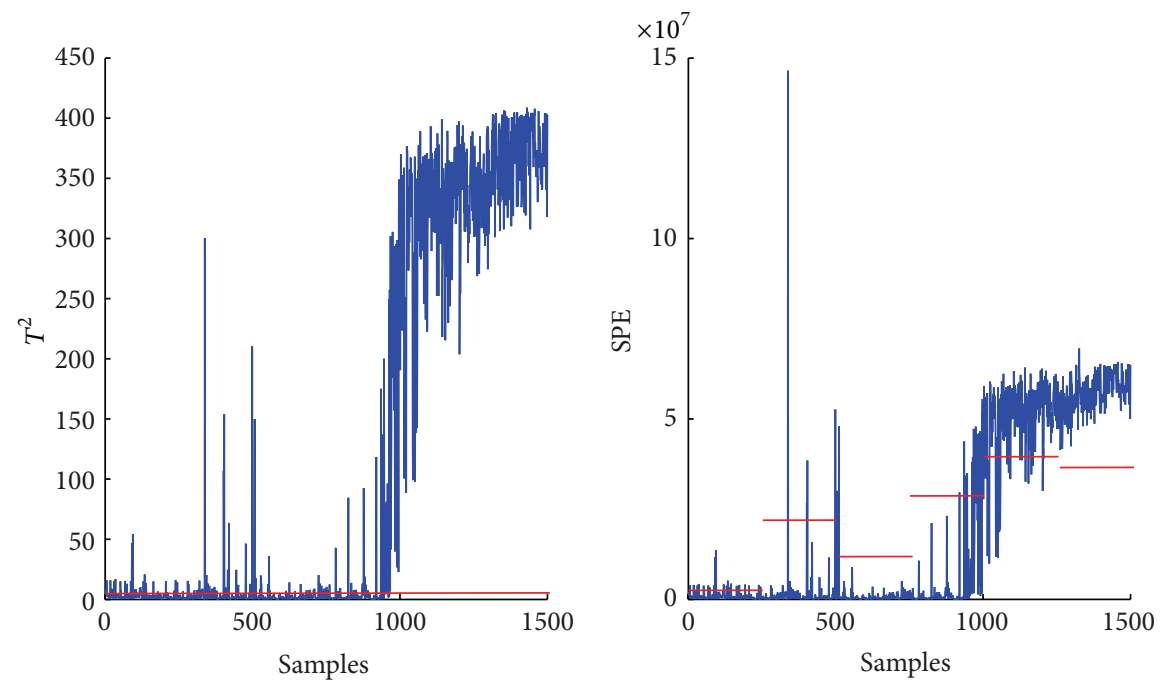

(b) Monitoring based on KPLS for fault 4
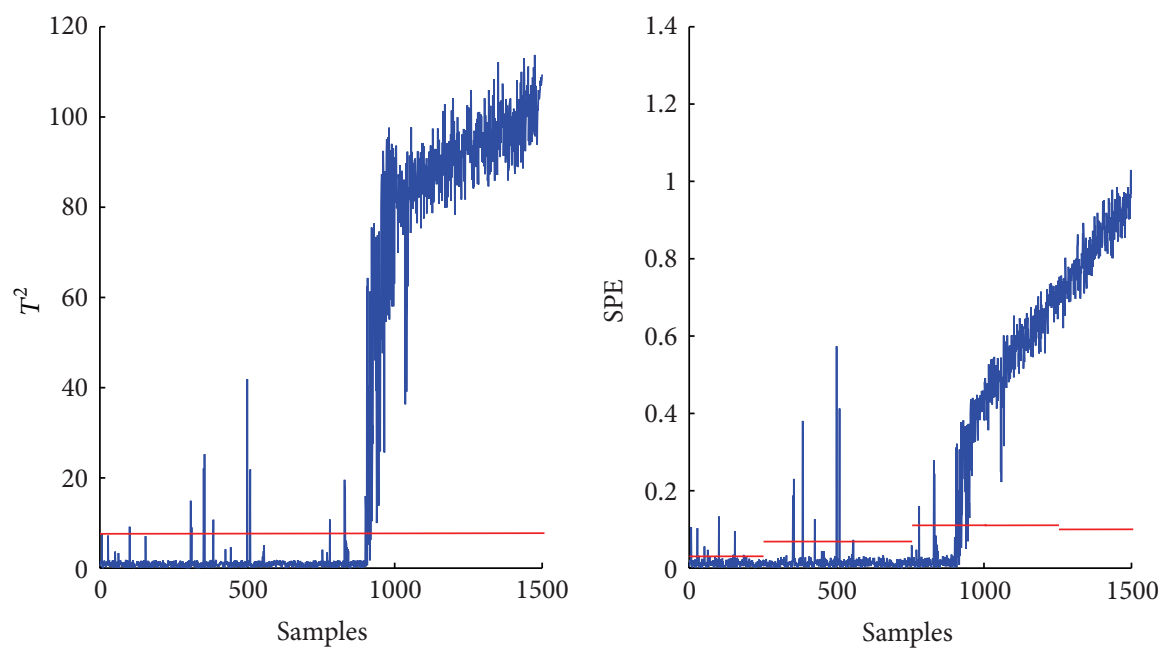

(c) Monitoring based on QKNPE for fault 4

FIgURE 6: Monitoring results for fault 4. 

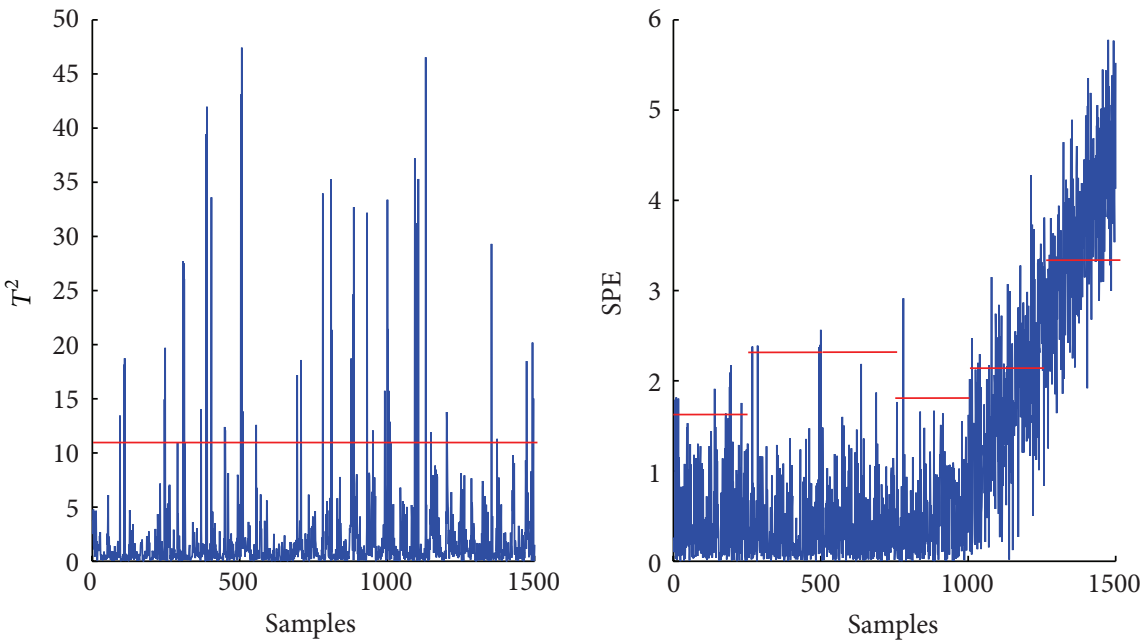

(a) Monitoring based on LLE for fault 5
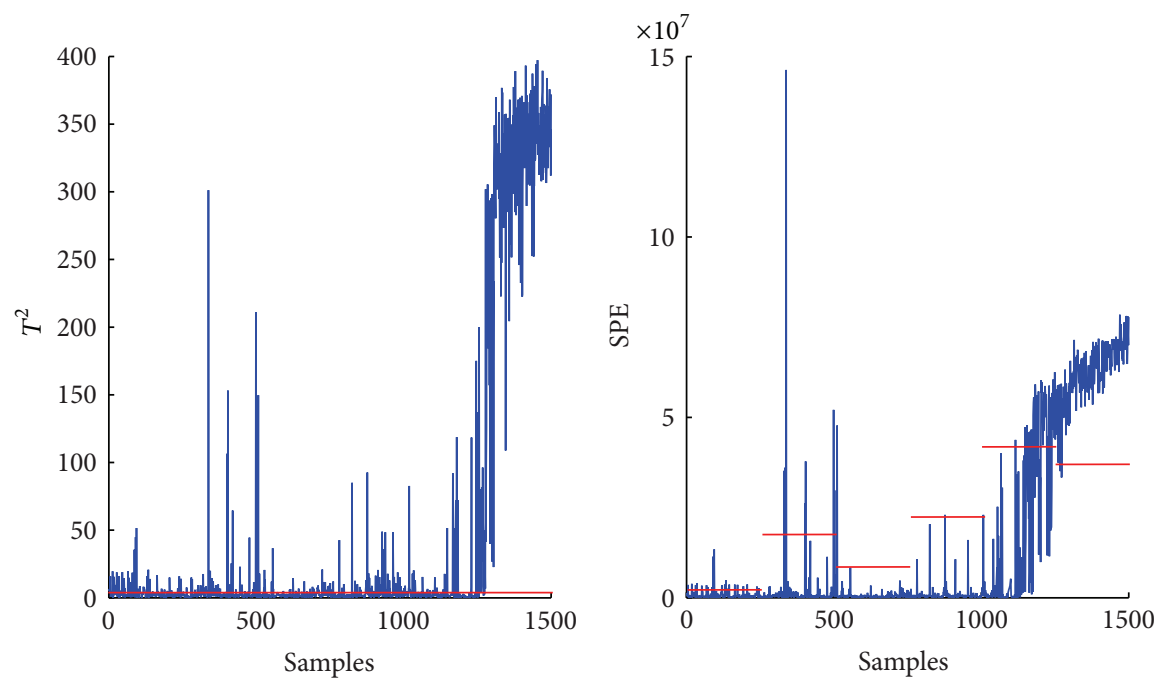

(b) Monitoring based on KPLS for fault 5
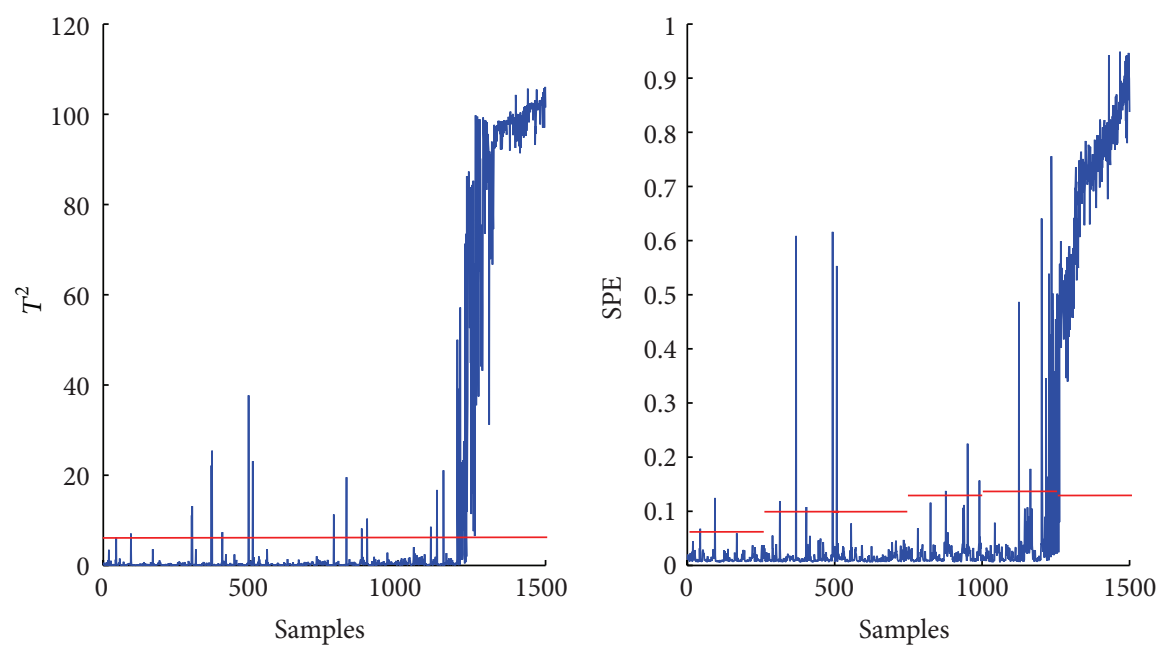

(c) Monitoring based on QKNPE for fault 5

FIgURE 7: Monitoring results for fault 5. 

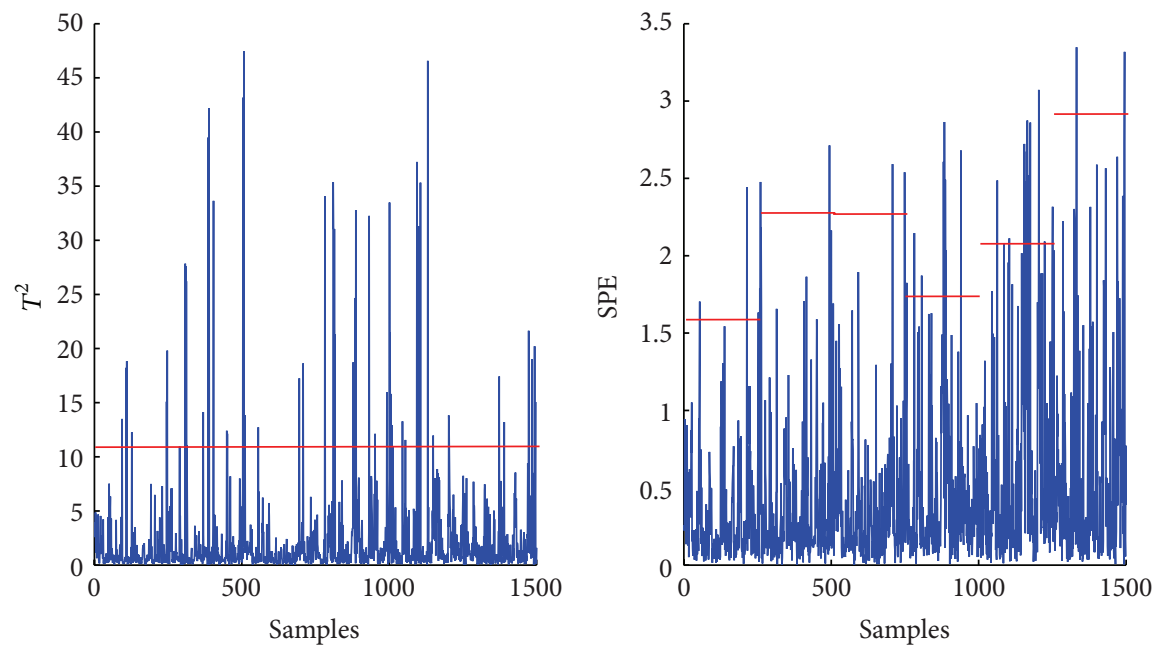

(a) Monitoring based on LLE for fault 6
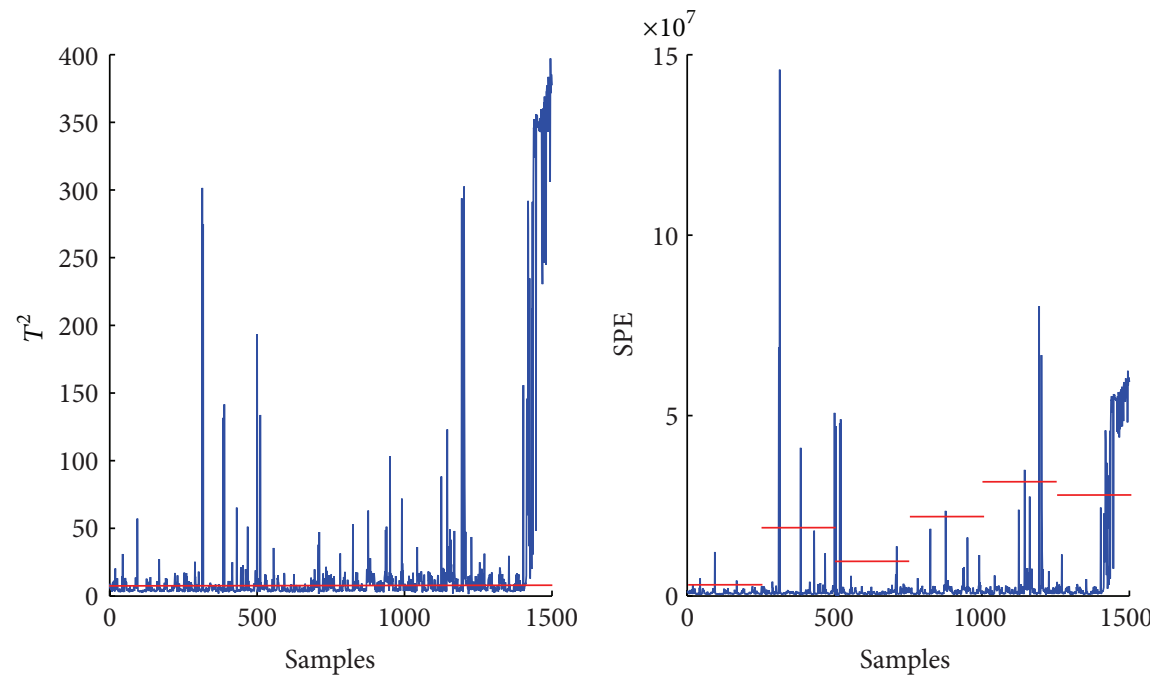

(b) Monitoring based on KPLS for fault 6
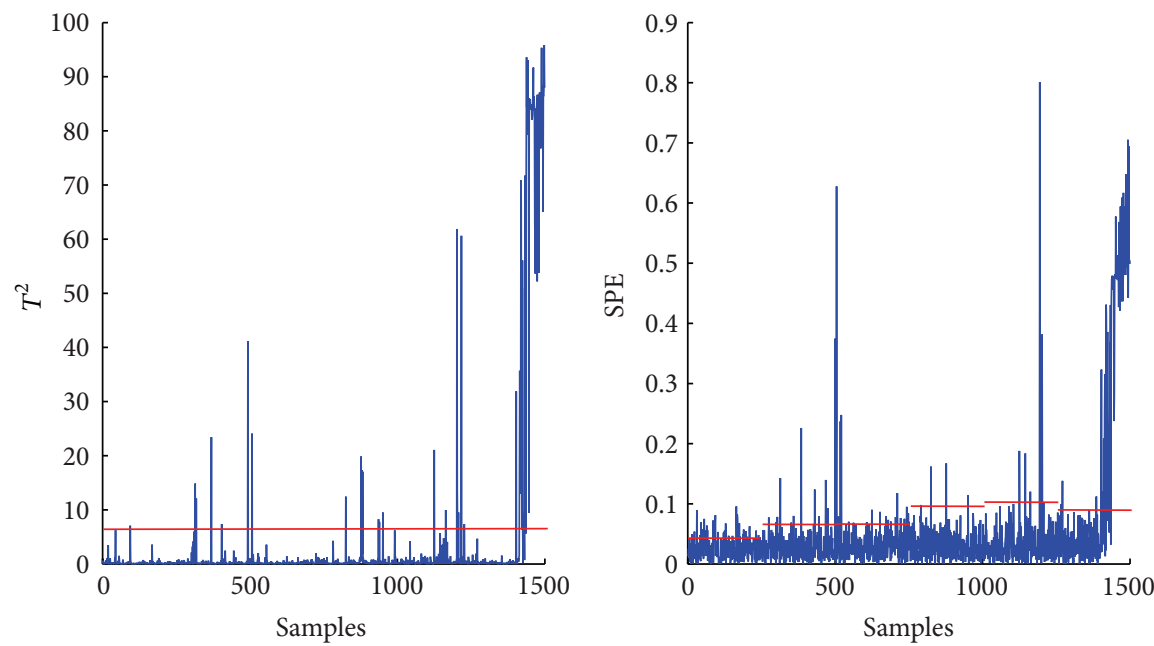

(c) Monitoring based on QKNPE for fault 6

FIgURE 8: Monitoring results for fault 6. 
TABLE 2: The monitoring accuracy rate, false alarm rate, missing alarm rate based on LLE, KPLS and QKNPE methods.

\begin{tabular}{|c|c|c|c|c|c|}
\hline Failure & Method & Statistics & Accuracy & Rate of false alarm & Rate of missing report \\
\hline \multirow{6}{*}{1} & \multirow{2}{*}{ LLE } & $T^{2}$ & 73.07 & 1.51 & 30.82 \\
\hline & & SPE & 99.60 & 3.02 & 0 \\
\hline & \multirow{2}{*}{ KPLS } & $T^{2}$ & 99.03 & 6.73 & 0 \\
\hline & & SPE & 98.93 & 5.13 & 0 \\
\hline & \multirow{2}{*}{ QKNPE } & $T^{2}$ & 99.93 & 0.50 & 0 \\
\hline & & SPE & 99.53 & 3.52 & 0 \\
\hline \multirow{6}{*}{2} & \multirow{2}{*}{ LLE } & $T^{2}$ & 99.40 & 2.01 & 0.09 \\
\hline & & SPE & 86.93 & 2.01 & 17.08 \\
\hline & \multirow{2}{*}{ KPLS } & $T^{2}$ & 97.40 & 6.22 & 0 \\
\hline & & SPE & 58.07 & 3.56 & 55.86 \\
\hline & \multirow{2}{*}{ QKNPE } & $T^{2}$ & 99.80 & 0.75 & 0 \\
\hline & & SPE & 99.07 & 3.26 & 0.09 \\
\hline \multirow{6}{*}{3} & \multirow{2}{*}{ LLE } & $T^{2}$ & 98.87 & 2.43 & 0 \\
\hline & & SPE & 61.60 & 1.29 & 70.79 \\
\hline & \multirow{2}{*}{ KPLS } & $T^{2}$ & 95.67 & 6.98 & 0 \\
\hline & & SPE & 96.45 & 2.30 & 4.57 \\
\hline & \multirow{2}{*}{ QKNPE } & $T^{2}$ & 99.60 & 0.86 & 0 \\
\hline & & SPE & 98.73 & 2.72 & 0 \\
\hline \multirow{6}{*}{4} & \multirow{2}{*}{ LLE } & $T^{2}$ & 63.00 & 3.23 & 87.52 \\
\hline & & SPE & 84.33 & 1.67 & 36.61 \\
\hline & \multirow{2}{*}{ KPLS } & $T^{2}$ & 91.07 & 8.42 & 8.65 \\
\hline & & SPE & 92.05 & 1.88 & 14.91 \\
\hline & \multirow{2}{*}{ QKNPE } & $T^{2}$ & 96.27 & 0.89 & 7.99 \\
\hline & & SPE & 95.07 & 2.34 & 8.82 \\
\hline \multirow{6}{*}{5} & \multirow{2}{*}{ LLE } & $T^{2}$ & 76.27 & 5.59 & 96.01 \\
\hline & & SPE & 86.87 & 1.50 & 59.47 \\
\hline & \multirow{2}{*}{ KPLS } & $T^{2}$ & 87.80 & 11.09 & 11.33 \\
\hline & & SPE & 92.93 & 2.13 & 18.24 \\
\hline & \multirow{2}{*}{ QKNPE } & $T^{2}$ & 96.87 & 1.25 & 10.63 \\
\hline & & SPE & 95.60 & 2.42 & 12.29 \\
\hline \multirow{6}{*}{6} & \multirow{2}{*}{ LLE } & $T^{2}$ & 88.53 & 5.58 & 93.07 \\
\hline & & SPE & 92.07 & 1.43 & 98.02 \\
\hline & \multirow{2}{*}{ KPLS } & $T^{2}$ & 87.42 & 11.19 & 16.41 \\
\hline & & SPE & 94.15 & 2.41 & 31.58 \\
\hline & \multirow{2}{*}{ QKNPE } & $T^{2}$ & 97.60 & 1.43 & 25.84 \\
\hline & & SPE & 96.20 & 2.64 & 19.80 \\
\hline
\end{tabular}

\section{Acknowledgment}

This work is supported by the National Natural Science Foundation (NNSF) of China under Grants 61325015 and 61273163.

\section{References}

[1] X.-G. Yan and C. Edwards, "Robust sliding mode observerbased actuator fault detection and isolation for a class of nonlinear systems," International Journal of Systems Science.
Principles and Applications of Systems and Integration, vol. 39, no. 4, pp. 349-359, 2008.

[2] Q. J. Wen, Z. Q. Ge, and Z. H. Song, "Data-based linear Gaussian state-space model for dynamic process monitoring," AIChE Journal, vol. 58, no. 12, pp. 3763-3776, 2012.

[3] Z. Q. Ge, Z. H. Song, and F. R. Gao, "Review of recent research on data-based process monitoring," Industrial \& Engineering Chemistry Research, vol. 52, no. 10, pp. 3543-3562, 2013.

[4] M. Kano and Y. Nakagawa, "Data-based process monitoring, process control, and quality improvement: recent developments and applications in steel industry," Computers and Chemical Engineering, vol. 32, no. 1-2, pp. 12-24, 2008. 
[5] J.-H. Cho, J.-M. Lee, S. W. Choi, D. Lee, and I.-B. Lee, "Fault identification for process monitoring using kernel principal component analysis," Chemical Engineering Science, vol. 60, no. 1, pp. 279-288, 2005.

[6] W. L. Du, Y. Tian, and F. Qian, "Monitoring for nonlinear multiple modes process based on LL-SVDD-MRDA," IEEE Transactions on Automation Science and Engineering, vol. 11, no. 4, pp. 1133-1148, 2014.

[7] Y.-S. Qi, P. Wang, and X.-J. Gao, "Fault detection and diagnosis of multiphase batch process based on kernel principal component analysis-principal component analysis," Control Theory \& Applications, vol. 29, no. 6, pp. 754-764, 2012.

[8] C. H. Zhao, F. R. Gao, and F. L. Wang, "Nonlinear batch process monitoring using phase-based kernel-independent component analysis-principal component analysis (KICA-PCA)," Industrial and Engineering Chemistry Research, vol. 48, no. 20, pp. 9163-9174, 2009.

[9] Y. Tian, W. L. Du, and F. Qian, "Fault detection and diagnosis for non-gaussian processes with periodic disturbance based on AMRA-ICA," Industrial and Engineering Chemistry Research, vol. 52, no. 34, pp. 12082-12107, 2013.

[10] Y. W. Zhang, "Fault detection and diagnosis of nonlinear processes using improved kernel independent component analysis (KICA) and Support Vector Machine (SVM)," Industrial \& Engineering Chemistry Research, vol. 47, no. 18, pp. 6961-6971, 2008.

[11] J. Dong, K. Zhang, Y. Huang, G. Li, and K. Peng, "Adaptive total PLS based quality-relevant process monitoring with application to the Tennessee Eastman process," Neurocomputing, vol. 154, pp. 77-85, 2015.

[12] Z. G. Zhao, Q. H. Li, M. Huang, and F. Liu, "Concurrent PLSbased process monitoring with incomplete input and quality measurements," Computers \& Chemical Engineering, vol. 67, pp. 69-82, 2014.

[13] D. Vyas and N. S. R. Krishnayya, "Estimating attributes of deciduous forest cover of a sanctuary in India utilizing Hyperion data and PLS analysis," International Journal of Remote Sensing, vol. 35, no. 9, pp. 3197-3218, 2014.

[14] S. Yin, X. P. Zhu, and O. Kaynak, "Improved PLS focused on key-performance-indicator-related fault diagnosis," IEEE Transactions on Industrial Electronics, vol. 62, no. 3, pp. 16511658, 2015.

[15] K. Kim, J.-M. Lee, and I.-B. Lee, "A novel multivariate regression approach based on kernel partial least squares with orthogonal signal correction," Chemometrics and Intelligent Laboratory Systems, vol. 79, no. 1-2, pp. 22-30, 2005.

[16] L. Wang and H. B. Shi, "Improved kernel PLS-based fault detection approach for nonlinear chemical processes," Chinese Journal of Chemical Engineering, vol. 22, no. 6, pp. 657-663, 2014.

[17] Y. W. Zhang and Z. Y. Hu, "Multivariate process monitoring and analysis based on multi-scale KPLS," Chemical Engineering Research \& Design, vol. 89, no. 12, pp. 2667-2678, 2011.

[18] J. L. Godoy, D. A. Zumoffen, J. R. Vega, and J. L. Marchetti, “New contributions to non-linear process monitoring through kernel partial least squares," Chemometrics and Intelligent Laboratory Systems, vol. 135, pp. 76-89, 2014.

[19] S. W. Choi, J. Morris, and I.-B. Lee, "Nonlinear multiscale modelling for fault detection and identification," Chemical Engineering Science, vol. 63, no. 8, pp. 2252-2266, 2008.
[20] Y. W. Zhang and S. Li, "Modeling and monitoring of nonlinear multi-mode processes," Control Engineering Practice, vol. 22, no. 1, pp. 194-204, 2014.

[21] S. J. Qin and Y. Y. Zheng, "Quality-relevant and processrelevant fault monitoring with concurrent projection to latent structures," AIChE Journal, vol. 59, no. 2, pp. 496-504, 2013.

[22] G. Li, S. J. Joe Qin, and D. H. Zhou, "Output relevant fault reconstruction and fault subspace extraction in total projection to latent structures models," Industrial and Engineering Chemistry Research, vol. 49, no. 19, pp. 9175-9183, 2010.

[23] G. Li, C. F. Alcala, S. J. Qin, and D. H. Zhou, "Generalized reconstruction-based contributions for output-relevant fault diagnosis with application to the Tennessee Eastman process," IEEE Transactions on Control Systems Technology, vol. 19, no. 5, pp. 1114-1127, 2011.

[24] Q. Liu, S. J. Qin, and T. Y. Chai, "Multiblock concurrent PLS for decentralized monitoring of continuous annealing processes," IEEE Transactions on Industrial Electronics, vol. 61, no. 11, pp. 6429-6437, 2014.

[25] S. M. Xiang, F. P. Nie, and C. S. Zhang, "Regression reformulations of LLE and LTSA with locally linear transformation," IEEE Transactions on Systems, Man, and Cybernetics Part B: Cybernetics, vol. 41, no. 5, pp. 1250-1262, 2011.

[26] Y. Goldberg, A. Zakai, D. Kushnir, and Y. Ritov, "Manifold learning: the price of normalization," Journal of Machine Learning Research, vol. 9, pp. 1909-1939, 2008.

[27] C. D. Tong and X. F. Yan, "Statistical process monitoring based on a multi-manifold projection algorithm," Chemometrics and Intelligent Laboratory Systems, vol. 130, pp. 20-28, 2014.

[28] X. H. Jin, F. Yuan, T. W. S. Chow, and M. B. Zhao, "Weighted local and global regressive mapping: a new manifold learning method for machine fault classification," Engineering Applications of Artificial Intelligence, vol. 30, pp. 118-128, 2014.

[29] X.-G. Yan and C. Edwards, "Nonlinear robust fault reconstruction and estimation using a sliding mode observer," Automatica, vol. 43, no. 9, pp. 1605-1614, 2007.

[30] X.-G. Yan and C. Edwards, "Adaptive sliding-mode-observerbased fault reconstruction for nonlinear systems with parametric uncertainties," IEEE Transactions on Industrial Electronics, vol. 55, no. 11, pp. 4029-4036, 2008.

[31] Y. W. Zhang, C. Wang, and R. Q. Lu, "Modeling and monitoring of multimode process based on subspace separation," Chemical Engineering Research and Design, vol. 91, no. 5, pp. 831-842, 2013.

[32] Y. W. Zhang, T. Y. Chai, Z. M. Li, and C. Yang, "Modeling and monitoring of dynamic processes," IEEE Transactions on Neural Networks and Learning Systems, vol. 23, no. 2, pp. 277-284, 2012.

[33] S. Mika, B. Schölkopf, A. Smola, K.-R. Müller, M. Scholz, and G. Rätsch, "Kernel PCA and de-noising in feature spaces," in Proceedings of the 12th Annual Conference on Neural Information Processing Systems (NIPS '98), pp. 536-542, December 1998.

[34] S. T. Roweis and L. K. Saul, "Nonlinear dimensionality reduction by locally linear embedding," Science, vol. 290, no. 5500, pp. 2323-2326, 2000. 


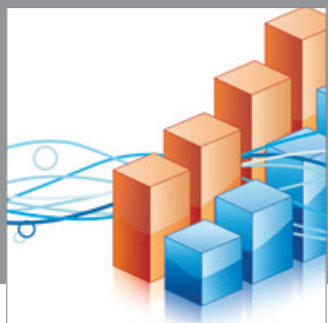

Advances in

Operations Research

mansans

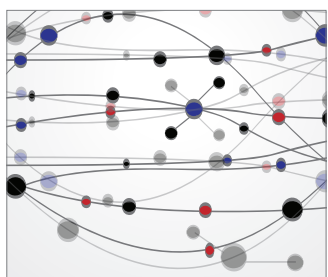

The Scientific World Journal
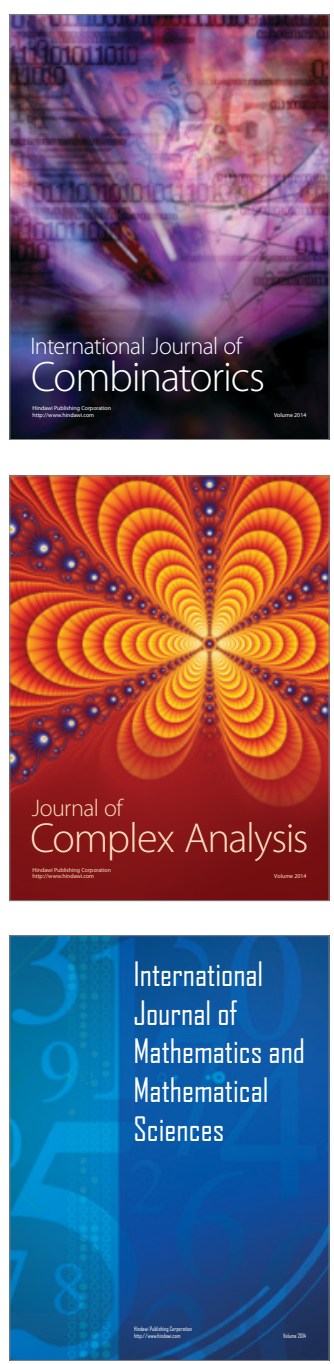
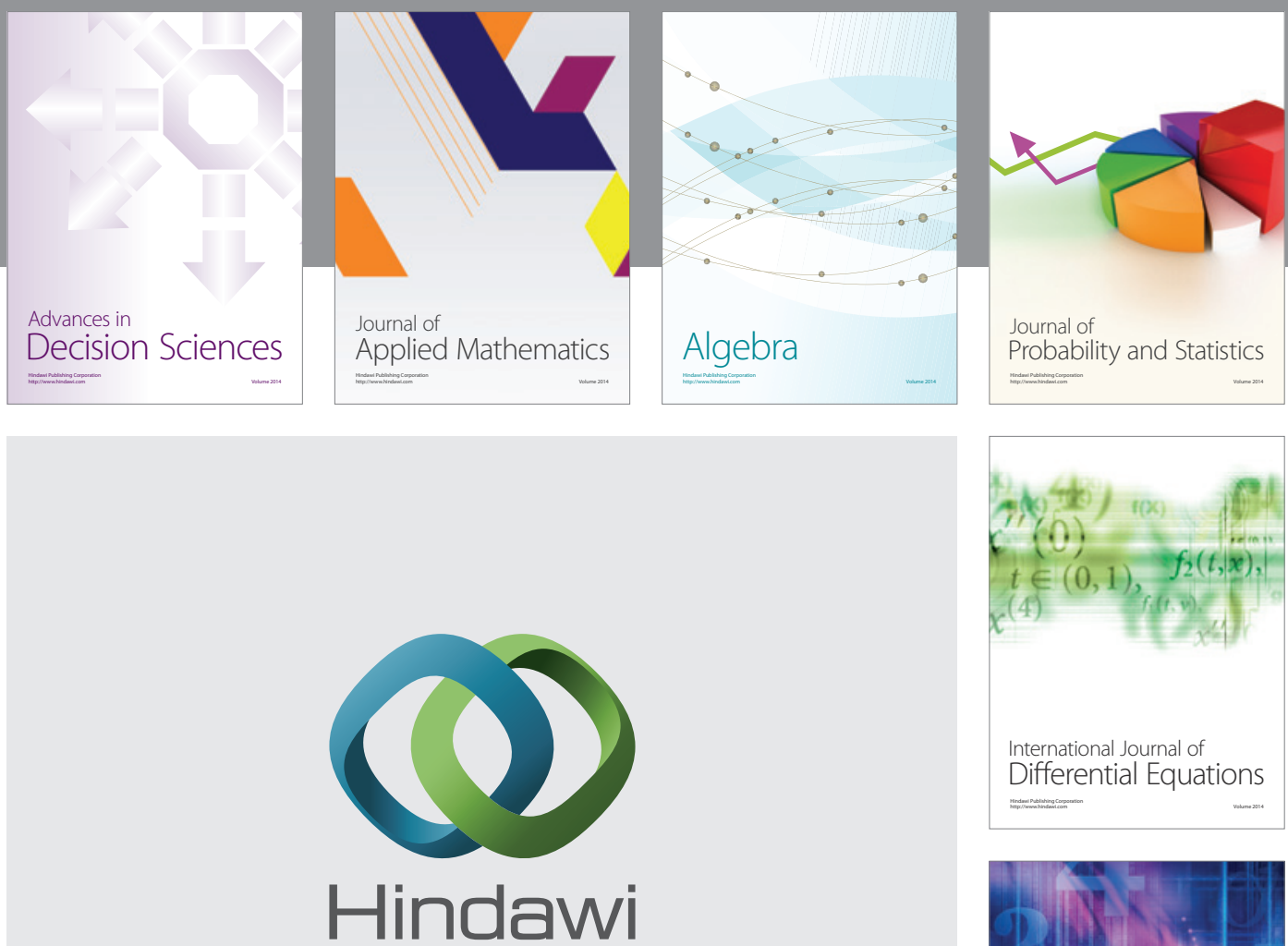

Submit your manuscripts at http://www.hindawi.com
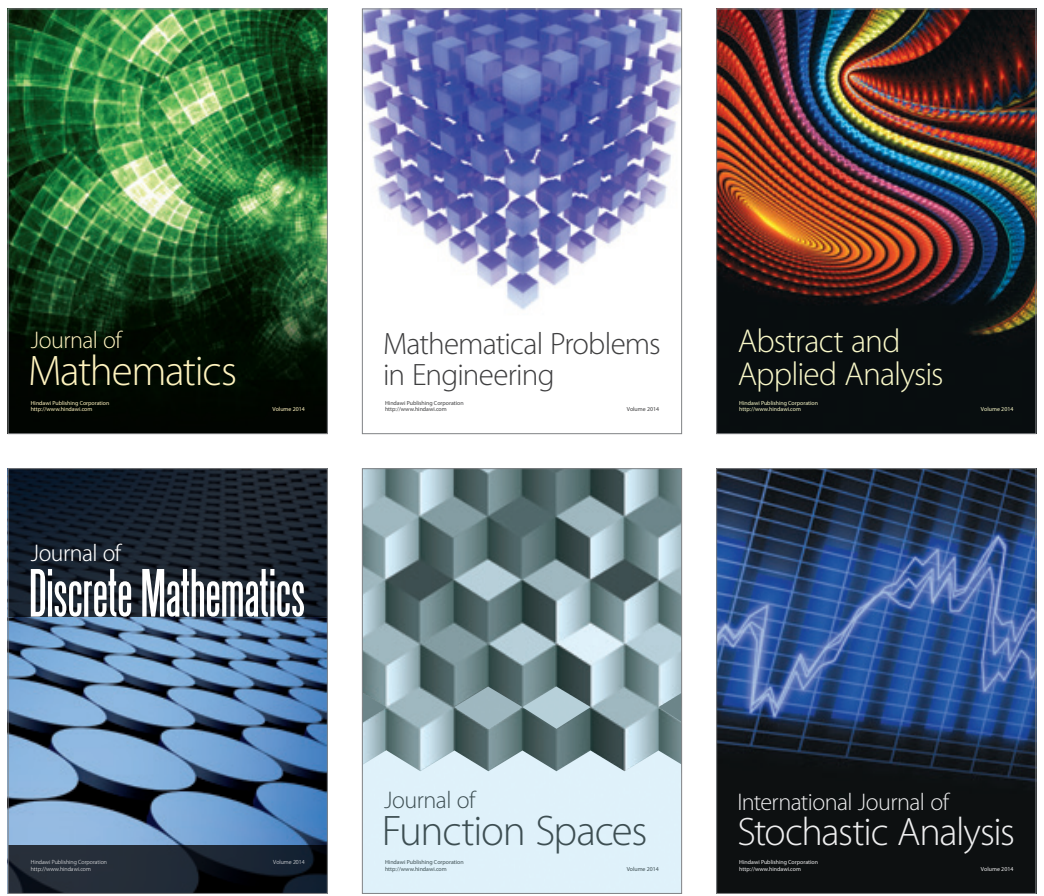

Journal of

Function Spaces

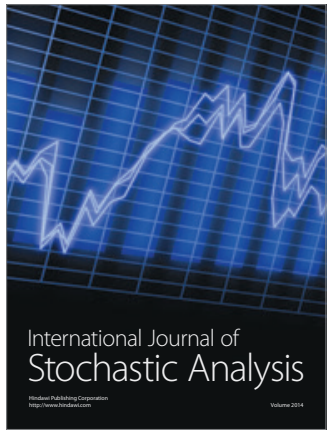

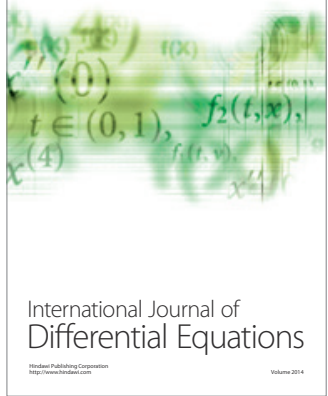
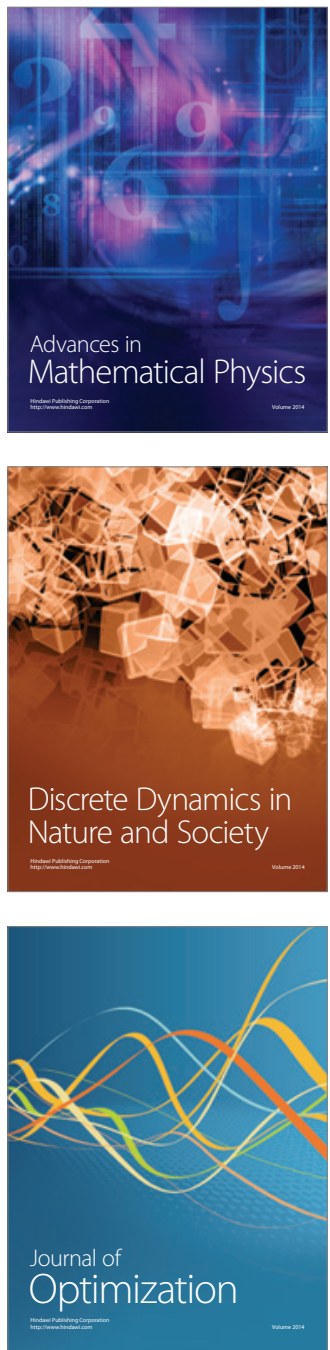